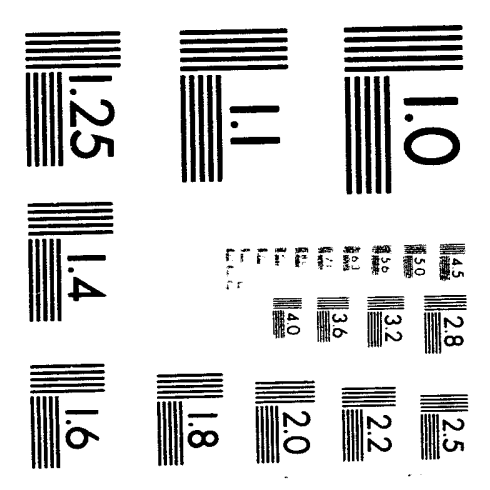



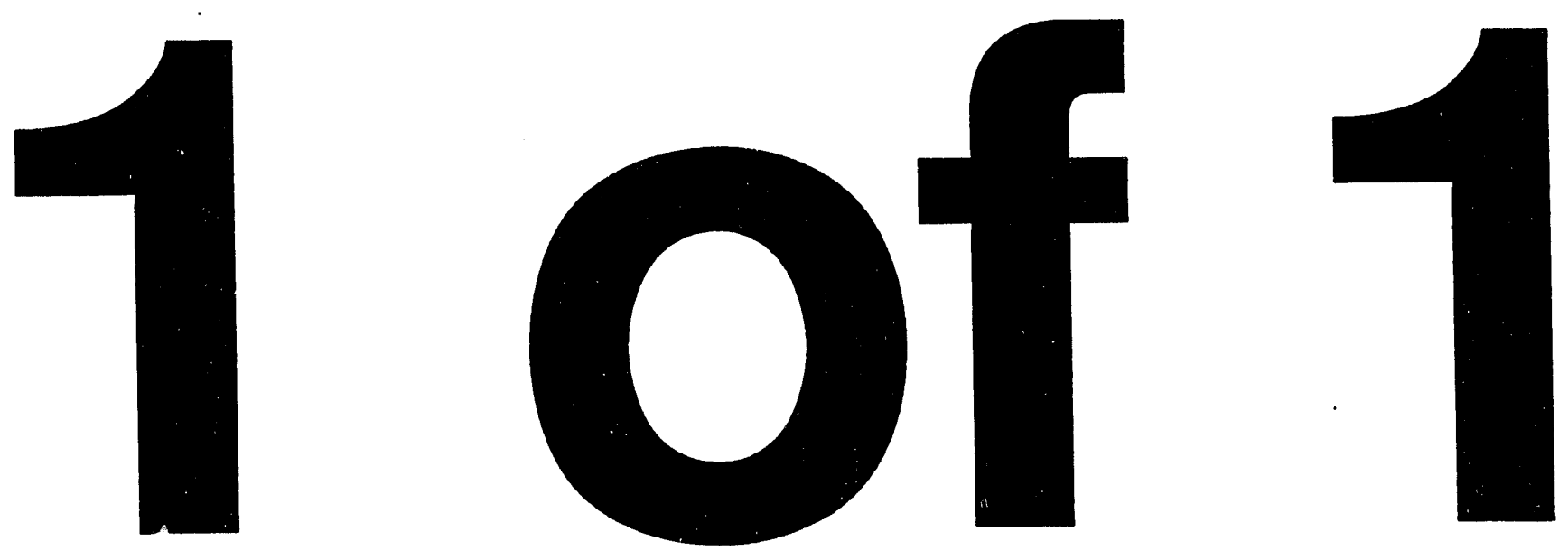


\title{
Electronic Tag and Position Sensor
}

\author{
Hughes Aircraft Company
}

January 20, 1992

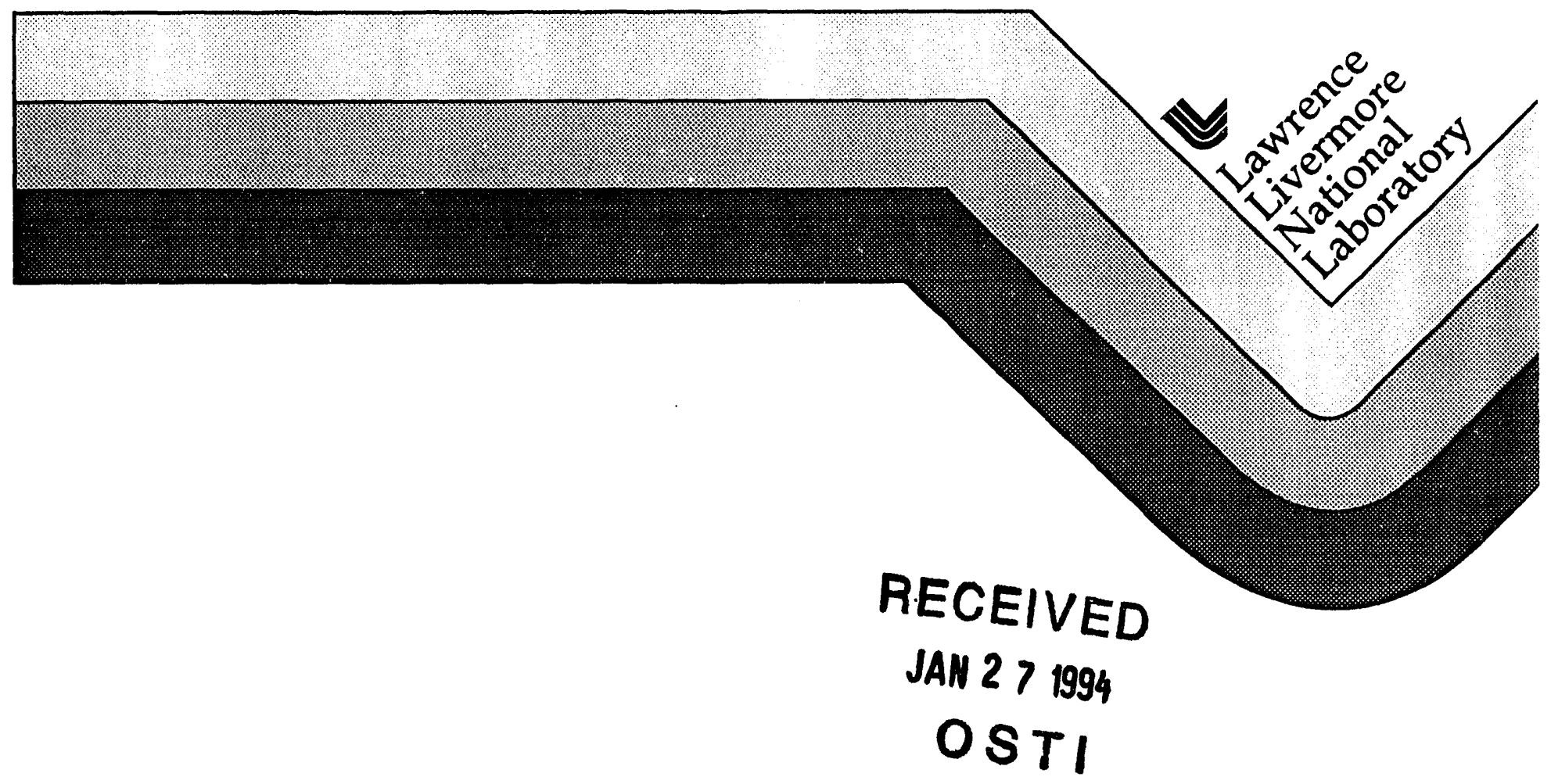




\section{MISCLAIMIER}

Work performed under the auspices of the L.S. Department of Fenergy by Lawrence Livermore National Laboratory under contract number $1 \$-7405-\mathrm{HN} ;-48$.

This document was prepared as an account of work sponsored by an agency of the Inited States Government. Neither the l'nited States Government nor the l niversity of California nor any of their employees, makes any warranty express or implied, or assumes any legal liability or responsibility for the accuracy, complefeness, or usefulness of any information, apparatus, product, or process disclosed, or represents that its use would not infringe privately owned rights. Reference herein to any specific commercial products, process, or service by trade name, trademark, manufacturer, or otherwise. does not necessarily constitute or imply its endorsement, recommendation, or favoring by the l'nited Siates Government or the Iniversity of California. I he views and opinions of authors expressed herein do not necessarily state or reflect those of the Inited States (iovernment or the I niversity of California. and shall not be used for advertising or product endorsement purposes. 
ELECTRONIC TAG AND POSITION SENSOR

Submitted January 20, 1992 by

\section{HUGHES AIRCRAFT COMPANY}

P. O. BOX 3310

FULLERTON, CA 92634

to

\section{UNIVERSITY OF CALIFORNIA}

\section{LAWRENCE LIVERMORE NATIONAL LABORATORY}

P. O. BOX 5012

7000 East Avenue

Livermore, CA 94551

Prepared under

Purchase Order B160443

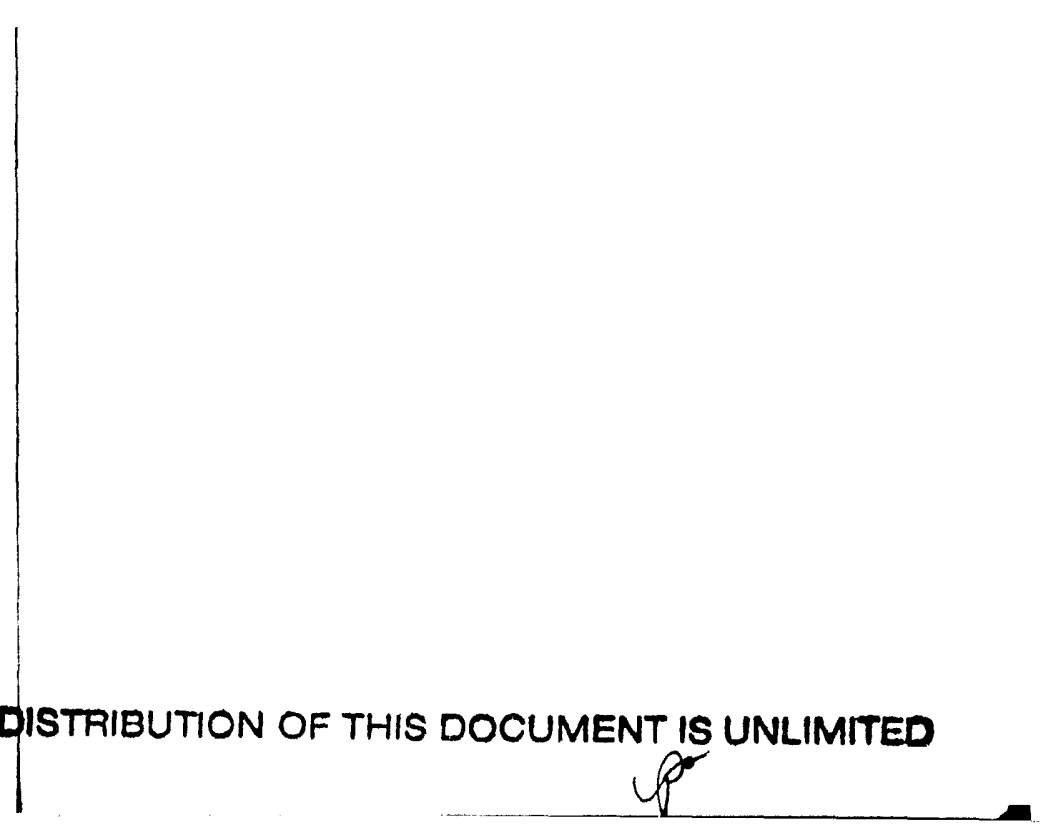




\section{Preface}

The following is the final report for P.O. B160443. The intent of this study phase program was to adequately define the Electronic Tag and Position Sensor chip so as to be able to price and schedule the full design and development culminating in a silicon IC. Therefore, even though Hughes Aircraft Company feels that the approach submitted in this document is what should be developed, it is still considered preliminary and could change as the full design is developed. 


\section{PROPOSED LLNL ELECTRONIC TAG AND POSITION SENSOR}

\section{PHYSICALFEATURES}

Single die containing the position sensor, encryption logic, crystal oscillator, and reader/tag communication/control logic fabricated in HUGHES EEPROM CMOS process.

Die size - estimated 300 to 350 mils 2 .

Packaging - TBD

Requirements are:

1) As small as possible.

2) Minimize height (from tagged surface) at expense of surface area.

\section{POWER}

\section{Power source}

The power source target requirement is $5 \mathrm{~V} \pm 20 \%$.

The digital logic in the tag will operate satisfactorily with $5 \mathrm{~V} \pm 20 \%$, but the analog circuits need a center ground supply of $\pm 5 \mathrm{~V}$. The proposed die will have 3 power pads $(+5 \mathrm{~V}, \mathrm{GND}$, and $-5 \mathrm{~V})$.

The packaged part will have either 2 or 3 power supply pins.

If only 2 power pins are used on the package, a converter chip will need to be added inside the package to provide the $-5 \mathrm{~V}$ supply.

\section{Power consumption}

Peak operating power target requirement is $<10 \mathrm{~mW}$.

Peak operating power is estimated at $<100 \mathrm{~mW}$ when the position sensor is operating. Peak operating power is estimated at $<10 \mathrm{~mW}$ for tag operations other than the position sensor.

\section{Sleep or low power mode}

For applications where power is constantly applied to the tag, the requirement is for a 10 year battery life at $60 \mu \mathrm{A}$ average current. An on chip sleep timer will stop the oscillator and disable power to the analog circuits after a period of 5 seconds of no activity on the data input line. In low power mode the only power consumption would be due to CMOS leakage current which is below the average current requirement. 


\section{INTERFACE}

\section{Data transmit and receive}

Data communication between tag and reader is asynchronous serial 8 bit ASCII format with no parity at 1200 or 9600 bits per second. The default rate for virgin tags is 9600 bits per second. The 1200 bit rate can be selected by secure command on initialized tags. Two package pins (or contacts) for data input/output are required. One pin is serial data input and the other is serial data output. It is required that the two data I/O pins be able to be tied together to form a single bi-directional line. The circuit configuration to do this is shown below. During data receive the internal TRANSMIT line will hold the data output buffer in high impedance mode while enabling the data receive circuit. During data transmit the internal TRANSMIT line will enable the data output buffer while disabling the data receive circuit.

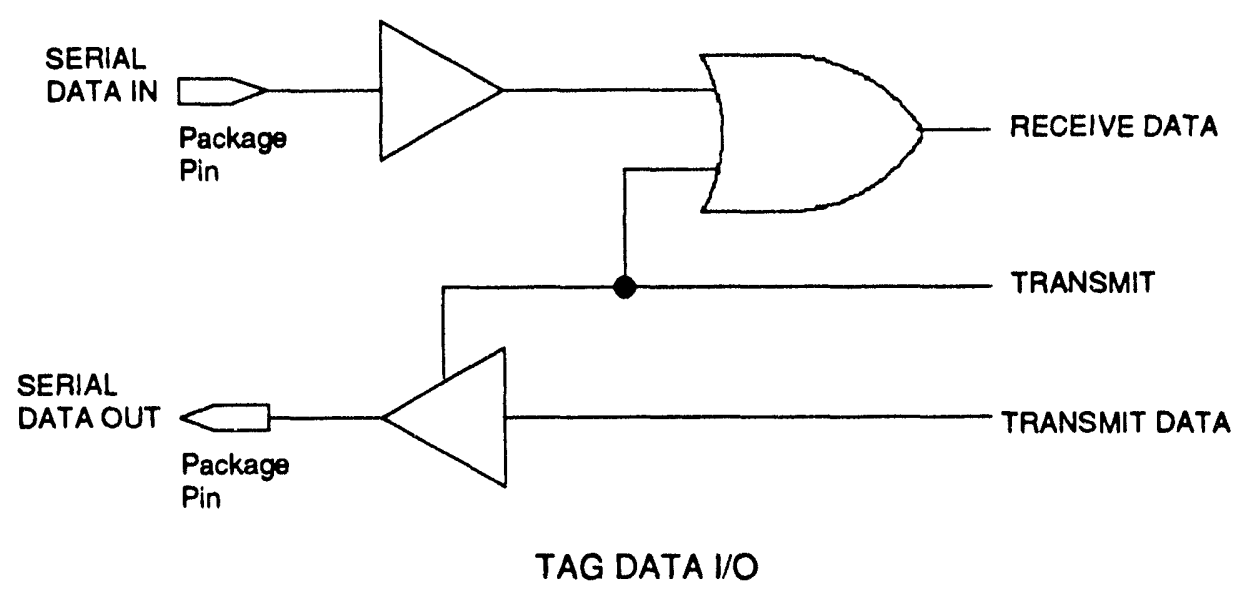

\section{Input ports}

Two digital input ports are available on the chip. They may or may not be connected to a digital signal or power source, depending on the package configuration or application. These inputs cannot be allowed to float when unconnected. If allowed to float, the CMOS input inverters will draw excessive current. Two possible configurations that will prevent these inputs from floating when unconnected are shown on the following page. Configuration $A$ will default to logic 1 on power up if the input is not connected. Configuration A will not draw current when driven with $\operatorname{logic} 1$ or logic 0 . Configuration $A$ if driven and then disconnected will retain the last driven state until power is turned off. Configuration B will always be logic 1 when not connected and will draw a small amount of current $(\sim 10 \mu \mathrm{A})$ when driven with a logic 0 . 


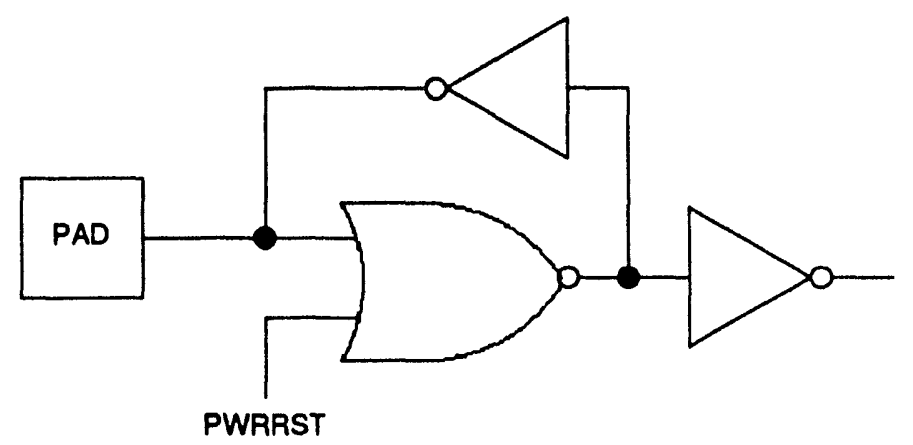

INPUT PORT (Conflguration A)

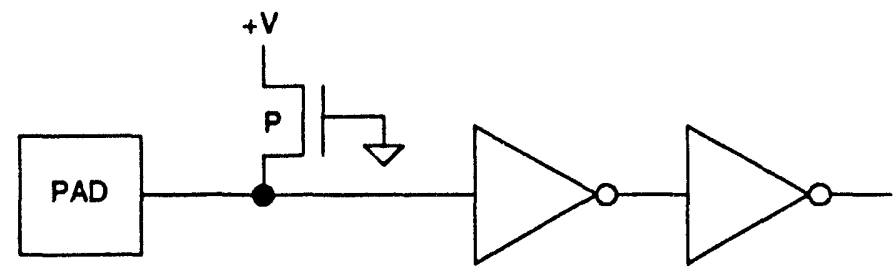

INPUT PORT (Configuration B)

\section{Qutput ports}

Two digital output ports are available on the chip. The state of each output port is changeable only by secure command. The state of each output port is stored in a nonvolatile bit. Default state of each port for a virgin tag is 1 .

\section{ENCRYPTION}

Encryption is triple DES (encrypt w/key1 - decrypt w/key2 - encrypt w/key3). Key storage is provided in EEPROM for 5 key sets. Each key set is composed of 3 keys. Each key is entered during tag initialization as 64 bits ( 16 hex characters). The DES algorithm uses only 56 bits of each key. In the DES every 8th bit is assumed to be a parity bit and is not used in the algorithm. DES does not do parity checking. The EEPROM stores only 56 bits for each key. A $120 \times 7$ EEPROM is used to store the key data.

The DES algorithm is implemented in hardware in the tag chip.

Requirement is for triple DES encryption $<.5$ second.

\section{OPERATION}

\section{Reader/tag communication}

The communication protocol for all reader/tag exchanges occurs in ASCII format. Only the printable ASCII characters are accepted as input to the tag. Depending on the operating mode, the tag may accept any printable (upper or lower case) character, only alpha (upper or lower case), only numeric, or only ASCII hex (upper case for alpha values). Characters of the wrong type for the tag mode of operation will cause the tag to generate the ASCII character "?" indicating an error and will reset the tag. The tag will output the carriage return and line feed characters as necessary. 


\section{Virgin tag detect}

Since a programmed tag can only be cleared by secure command, a tag after manufacture must be guaranteed to be in a virgin state without any external way of clearing it (otherwise it could never be tested or initialized). The contents of nonvolatile memory after manufacture are random. There is no guarantee that it will be in the all zeros or all ones state. Sixteen nonvolatile bits are used to indicate the virgin status of the tag. A single 16 bit pattern such as 01101010110101 indicates a nonvirgin or initialized tag. All other patterns of which there are $2^{16}-1$ will indicate a virgin tag. The probability of these 16 nonvolatile bits indicating a virgin tag after manufacture is $1-2^{-16}$ or 0.99998 . After a proper initialization is performed this 16 bit pattern is set to the nonvirgin pattern and written to the nonvolatile bits, which can then only be cleared to all zeros by secure command.

\section{Initialization mode}

When power is applied to the tag and the virgin status indicates a virgin tag, the tag enters the initialize mode of operation. The tag will erase (set to zerc) all nonvolatile bits. The tag will then output the text prompts in the order shown in the following table. Each text prompt is preceded by a cr/f. After outputting a text prompt, the tag will wait for a proper input response before outputting the next text prompt. As proper characters are entered for each prompt they are stored in the ID_DATA EEPROM or the KEY_DATA EEPROM. Improper (not expected input) characters will cause the tag to output the ASCII character "?" indicating an error and restart initialize mode. If the check sum entered is correct, the tag will store the 16 bit nonvirgin code in the virgin nonvolatile status bits and go into interrogate mode. If the check sum entered is not correct, the tag will erase all nonvolatile bits and restart initialize mode. The check sum is calculated on the 8 bit ASCII input .

The following table contains suggested spellings for the tag output prompts. The spellings are designed to convey the desired information using a minimal number of characters without being too cryptic. 


\section{INITIALIZATION MODE OUTPUT/INPUT TABLE}

\begin{tabular}{|c|c|}
\hline TAG OUTPUT & EXPECTED INPUT \\
\hline cr/lf TAGID: & 6 ASCII - 1st character alpha, next 5 numeric \\
\hline $\mathrm{cr} / \mathrm{f}$ KEYID: & 6 ASCII - any printable character \\
\hline cr/f PARAM: & 3 ASCII - any printable character \\
\hline cr/ff VERSION: & 1 ASCII hex character - uppercase alpha \\
\hline cr/ff KEYSET0: & 16 ASCII hex characters - uppercase alpha \\
\hline $\mathrm{cr} / \mathrm{f}$ & 16 ASCI hex characters - uppercase alpha \\
\hline $\mathrm{cr} / \mathrm{lf}$ & 16 ASCII hex characters - uppercase alpha \\
\hline cr/ff KEYSET $1:$ & 16 ASCII hex characters - uppercase alpha \\
\hline $\mathrm{cr} / \mathrm{ff}$ & 16 ASCII hex characters - uppercase alpha \\
\hline $\mathrm{cr} / \mathrm{ff}$ & 16 ASCII hex characters - uppercase alpha \\
\hline cr/ff KEYSET2: & 16 ASCII hex characters - uppercase alpha \\
\hline $\mathrm{cr} / \mathrm{ff}$ & 16 ASCII hex characters - uppercase alpha \\
\hline $\mathrm{cr} / \mathrm{ff}$ & 16 ASCII hex characters - uppercase alpha \\
\hline cr/ff KEYSET3: & 16 ASCII hex characters - uppercase alpha \\
\hline $\mathrm{cr} / \mathrm{ff}$ & 16 ASCII hex characters - uppercase alpha \\
\hline $\mathrm{cr} / \mathrm{lf}$ & 16 ASCII hex characters - uppercase alpha \\
\hline cr/lf KEYSET4: & 16 ASCII hex characters - uppercase alpha \\
\hline $\mathrm{cr} / \mathrm{hf}$ & 16 ASCII hex characters - uppercase alpha \\
\hline $\mathrm{cr} / \mathrm{lf}$ & 16 ASCII hex characters - uppercase alpha \\
\hline cr/If CHEKSUM: & 2 ASCII hex characters - uppercase alpha \\
\hline
\end{tabular}

\section{Interrogation mode}

An initialized tag will enter interrogation mode upon power being applied or upon being awakened from sleep mode and will initiate communication hy identifying itself and requesting stimulus. The tag will then idle for a limited ume (sleep timeout) awaiting a stimulus from the reader. If no activity occurs on the data input line during the sleep time-out period, the tag will enter the sleep mode until awakened by the first character entered on the data input line and then identify itself again and await further input from the reader. Any character on the data input line will awaken a sleeping tag. The character used to awaken a sleeping tag is not processed as input stimulus. Upon receiving a proper stimulus, the tag responds with the requested information. As in the initialization mode, the tag will output the ASCII character "?" indicating an error and reset when an improper character is received.

The tag will identify itself by outputting "cr/f TAGID: Annnnn " where "Annnnn" is the 6 character ASCII TAGID.

The tag w'ill request stimulus by outputting "cr/f STIMULUS: ".

During stimulus input, if more than 5 seconds elapse between character entries, the tag will reset and enter the sleep mode. 


\section{Tagstimulus response}

A stimulus is composed of two distinct parts:

1) A single hex digit denoting key/function.

0 thru 2 for authentication requests, and

$\mathrm{A}$ thru $\mathrm{C}$ for special function requests.

2) Parameters associated with the specific key/function.

For example, a sixteen hex digit challenge for authentication requests.

The order of character input and output as shown in the table below is from left to right as it would appear on a terminal screen. The leftmost digits are considered the high order digits while the rightmost digits are considered the low order digits. Input and output are therefore high order characters first, low order characters last.

$V$ denotes clear text ASCII or hex digit

$X$ denotes ASCII hex digit

$Y$ denotes encrypted ASCII hex digit

$Z$ denotes check sum ASCII hex digit

\begin{tabular}{|c|c|c|c|}
\hline STIMULUS & TAG RESPONSE & COMMENT & NOTE \\
\hline OXXXXXXXXXXXXXXXXX & YYYYYYYYYYYYYYYYZZ & use key_set 0 & $\# 1$ \\
\hline IXXXXXXXXXXXXXXXXX & YYYYYYYYYYYYYYYYZZ & use key_set 1 & $\# 1$ \\
\hline $2 X X X X X X X X X X X X X X X X$ & YYYYYYYYYYYYYYYYZZ & use key_set 2 & $\# 1$ \\
\hline AXXXXXXXXXXXXXXXXXX & $\begin{array}{l}\text { ACKNOWLEDGE or } \\
\text { YYYYYYYYYYYYYYYYZZ }\end{array}$ & use key_set 3 & $\# 2$ \\
\hline B & YYYYYYYYYYYYYYYYZZ & use key_set 4 & $\# 3$ \\
\hline C & VVVVVVVVVVVVVVVVVZZ & no encryption & $\# 4$ \\
\hline
\end{tabular}




\section{NOTES RELATED TO TAG STIMULUS RESPONSE}

Note \#1: Response is encrypted value of stimulus.

Note \#2: Secure commands. After encrypting the stimulus using key_set 3:

If the high order encrypted 10 hex digits are all 0 's, and:

If the next 4 hex digits equal Counter_3, and:

If the last 2 hex digits are $X X$, execute specified secure command " $X X$ ".

Response is: ACKNOWLEDGE, and Counter_3 is advanced.

Else: Output the encrypted stimulus with a check sum.

Note \#3: Tamper sensor information before encryption.

Char 00-01: Check sum on encrypted ASCII output (2 hex digits).

Char 02: Input/Output port status.

bit 0: Output port 0 .

bit 1: Output port 1.

bit 2: Input port 0.

bit 3: Input port 1.

Char 03-05: Tamper sensor reading (3 hex digits).

Char 06-07: RAM parameter (2 hex digits).

Char 08-13: Counter_2 value (6 hex digits).

Char 14-17: Counter_1 value (4 hex digits).

Note \#4: Key control and feature identification. Clear text (never encrypted).

Char 00-01: Check sum on ASCII output (2 hex digits).

Char 02-07: KEYID (6 ASCII characters).

Char 08-10: PARAM (3 ASCII characters).

Char 11: VERSION (1 ASCII character) - software version.

Char 12-13: Mode status (2 hex digits) - not used (default value 00).

Char 14-17: Counter_3 value (4 hex digits).

\section{Counters associated with tag operations}

Counter_1 (16 bit EEPROM) is advanced each time tag is powered on.

Counter_2 (24 bit RAM) is advanced once per request for tamper sensor number.

Counter_3 (16 bit EEPROM) is advanced each time a secure command is acknowledged. 


\section{Secure commands}

A stimulus beginning with hex $A$ followed by 16 hex digits is a secure command stimulus. The tag encrypts the 16 hex digits with the triple DES algorithm using key_set 3 and then looks at the result. If the high order encrypted 10 hex digits are all 0 's and the next 4 hex digits equal Counter_ 3 and the last 2 hex digits equal a secure command code, then the tag will execute the secure command and output ACKNOWLEDGE. Otherwise the tag will output the encrypted result with a check sum. The eleven secure commands and possible hex codes are shown in the table below.

\section{SECURE COMMANDS}

\begin{tabular}{|c|l|}
\hline HEX & \multicolumn{1}{c|}{ COMMAND FUNCTION } \\
\hline 00 & Set output port 0 to 0. \\
\hline 11 & Set output port 1 to 0. \\
\hline 22 & Set output port 0 to 1 (default). \\
\hline 33 & Set output port 1 to 1 (default). \\
\hline 44 & Set baud rate to 1200. \\
\hline 55 & Set baud rate to 9600 (default). \\
\hline 66 & LOCK_ENABLE - Set lock enable bit to 1. \\
\hline 77 & LOCK_DISABLE - Set lock enable bit to 0 (default). \\
\hline 88 & CLEAR - Set tag to virgin state. \\
\hline 99 & UNLOCK - Unlock tag for 10 input attempts. \\
\hline AA & RAM_PARAM - Set RAM parameter to hex AA (default $=00$ ) \\
& and reset Counter_1. \\
\hline
\end{tabular}

\section{Lock mode}

If the lock enable bit is set to 1 , the tag will go into lock mode on power up or after the next 10 input attempts. When in locked mode, the tag will only respond to the " $C$ " input stimulus (clear text output) or the UNLOCK command. The UNLOCK command will unlock the tag for 10 input attempts. The UNLOCK command will not change the lock enable bit. The lock enable bit is a nonvolatile bit and can only be changed by secure command. Default state of the lock enable bit for a virgin tag is 0 .

\section{Baud rate}

The baud rate used for reader/tag communication is selectable by secure command for 1200 or 9600 bits per second. A nonvolatile bit is used to store the baud rate status. A value of 0 indicates 9600 baud while a value of 1 indicates 1200 baud. The default baud rate for a virgin tag is 9600 .

\section{BAM parameter}

The RAM parameter is an 8 bit code stored in volatile memory. On power up the RAM parameter will always have a value of all 0's. The only other value of the RAM parameter is the hex value AA, which is set only by secure command. 


\section{Mode status}

Mode status ( 2 hex digits) is not defined and unless otherwise specified will default to 00 .

\section{Power on reset}

The chip will have a RESET input pad that will be connected to an external capacitor inside the package to provide the power-on reset function. The RESET pad will only be bonded to a package pin for test packages.

\section{TESTMODE}

Test mode will be incapable of divulging keys or nonencrypted sensor data.

Test mode may contain one or more of the following features:

- Test pad on die for wafer test.

- Test mode entered by unique combination of inputs or an input configuration not used in normal operation.

- Test pin on package for packaged part test.

- Set scan for non sensitive portion of circuit.

- Test mode enabled when tag is virgin and disabled by either secure command or when tag is initialized.

\section{CONNOISSEUR COATING}

The die will be coated with a connoisseur coating to conceal as many of the critical nodes as possible. This will enhance the tamper resistance of the chip. The coating prevents the top surface of the IC from being visually inspected or physically probed.

As much of the circuitry as possible is put on the same surface of the IC to maximize the efficacy of the coating. The proposed system will have all of the analog and digital circuitry and the driven sensors on the top surface of the IC. Although the bulk of the digital circuitry can be kept to small enough geometries that the connoisseur coating protects them, some of the analog circuitry by its very nature will have nodes large enough that they can be probed externally. The IC does a noncontact capacitance measurement which will change if the coating is removed near the analog portions of the IC. This design feature will make probing the analog sections of the IC useless. 


\section{POSITION SENSOR DESCRIPTION}

\section{Number of transmitting electrodes}

The analog portion of the sensor block is shown in Figure 4. The chip uses multiple electrodes to quantify a position. The number of electrodes is set at 4 to allow the electrode size to be fairly big while still having enough electrodes to have many possible pairs of transmitting electrodes. The electrodes measure the rough orientation of the edge of the fiducial to the edge of the IC when there is an edge of the fiducial across the electrode face. It appears that increasing the number of electrodes beyond 4 will not add significant information because the adjacent electrodes will give almost the same readings but with degraded accuracy.

\section{Transmit electrodes and capacitors}

The electrodes used to transmit the electric field and the capacitors used in different parts of the circuit are large analog structures (The electrodes are approximately 100 mils on a side). Although they can be probed through the connoisseur coating, it is presently thought that there is not enough information on these nodes to make it worthwhile to further protect them from external tampering or probing. There are some additional structures which could be added to the chip if there is a compelling reason to determine if there has been an attempt to tamper with or probe these nodes.

These additional structures which comprise a tamper sensor are shown in Figure 1. Here each capacitor is surrounded, above and below, by a conducting layer. The conducting layers are designed to be at the minimum width and spacing allowed by the processing and is one long serpentine structure. The part will do an approximate resistance measurement of this line either before or after it measures the present position to detect possible tampering. If the line above or below the electrode plate is broken, then this could be detected and a bit set in the EEPROM to indicate that the chip has been tampered with. The electrodes on the front side are also coated by a dielectric (connoisseur coating). If the coating is partially removed it will perturb the sensor readings, so that any measurements taken afterwards are fairly meaningless.

\section{Pickup electrode}

The pickup electrode is presently planned to be external to the IC. The pickup electrode has a much smaller signal level on it if it is physically on the IC (about a factor of 500 lower). As shown in Figure 2 the signal is reduced because the capacitance between the pickup electrode and the IC will be 500 times larger than the capacitance between the pickup electrode and the fiducial.

The placement of the pickup electrode on the exterior of the IC means that it can be easily probed and monitored by someone attempting countermeasures. The IC has been designed to minimize the amount of useful information discernable from this point. The 
information on this node provides a minimum of information since the electronics on the chip scramble the applied electric fields which couple into the fiducial. A rough top view of the proposed assembly is shown below (not to scale).

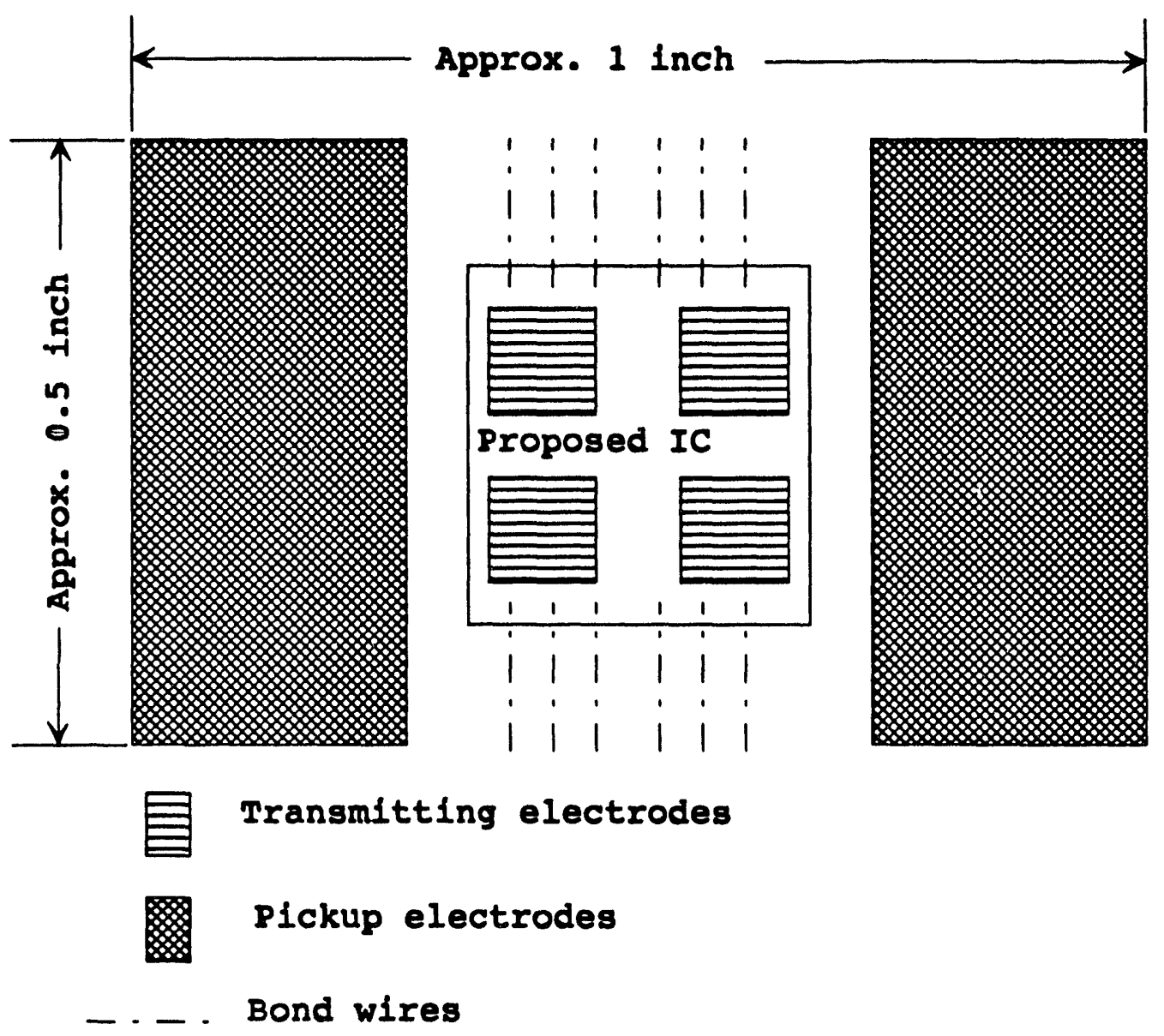

\section{Position measurement electronics}

The IC has a total of 4 transmitting electrodes. The transmitting electrodes are approximately 100 mils on a side and are located in each corner of the IC. These electrodes couple into the pickup electrode via the external fiducial to measure their relative position to the fiducial. A rough schematic to illustrate the measurement technique is shown in Figure 3. The circuitry inside the dashed line is internal to the IC. The fiducial and the pickup electrode are external to the IC. The transmitting electrodes form an array. If the fiducial is a line or similar structure the resultant position dependent information will depend not only upon the $\mathrm{x}$ and $\mathrm{y}$ displacement of the array compared to the fiducial but also the angular orientation of the array compared to the fiducial. This will further thwart tampering. One side effect of this technique is an increase in the number of position dependent bits. The position sensor reading must be changed from the 
originally proposed format to accommodate a digital word of more than 12 bits to represent the output data.

\section{The position sensing algorithm}

The basic algorithm that will be implemented is first described for a simplified hypothetical sensor which uses a straightforward measurement procedure. The algorithm will find a position dependent set of values for the sensor.

The simplest method is to apply a voltage to only one transmitting electrode at a time. The signal to be applied to the electrode is a square wave which cycles between the power supplies applied to the chip. This will maximize the available signal on the pickup electrode. In the simplest implementation, DAC1 and DAC2 shown in Figure 3, are just switches to the supplies. At the receiving end, the amount of charge coupled into the pickup electrode is measured either by nulling the output of the input amp by driving the capacitor Cin by DAC3 or by measuring the output of the input amp with an A/D.

The net result is the capacitance of $\mathrm{Ctlf}$ :

$$
\mathrm{Ct} 1 \mathrm{f}=\frac{\text { Vout }(\mathrm{D} / \mathrm{A} 3)^{*} \mathrm{Cin}}{\text { Vsupply }} \text { or } \mathrm{Ct} 1 \mathrm{f}=\frac{\text { Vout (Input amp }) * \mathrm{Cf}}{\text { Vsupply }}
$$

The different capacitances between the different transmitting electrodes to the fiducial can be measured sequentially to get a set of capacitances for a position dependent value. This would be used to detect movement of the chip relative to the fiducial. The expected size of the electrodes is $100 \mathrm{mils}$ ( 2540 microns) on a side. To get a resolution of better than 1 micron will require the resulting signal to be digitized by a 12 bit $A / D$, which is well within the capabilities of the CMOS process. Because of the small size of the original signal (on the order of 4 picocoulombs) there will be substantial noise which will be filtered out by averaging over several samples.

The position sensor described above has several advantages to it in its simplicity. For the proposed use it has one major drawback. The part can be fooled into reading any position that someone may want it to read, by probing and then driving the pickup electrode .

There are two main approaches to use in order to make the sensor reading more secure. One is to scramble the applied voltages and how they are sequenced such that it is not known by the outside world what the applied response is which gave the present response. This is the approach which is being incorporated by the proposed IC. In addition, the proposed chip will alter the sequence each time to further reduce any information which can be discerned from the outside world. The second approach is to further hide the pickup electrode. The natural location to hide the pickup electrode is to have an additional electrode on the surface of the IC. The problem with this approach is the massive reduction of the signal coupled back into the IC. This reduction is large 
enough that it would be very hard to design the circuitry to do an accurate position sensor due to noise, time and size constraints.

The scrambling of the data is done by having a large set of possible stimuli on the transmitting electrodes and the respective portions unscrambled at the receiving end. The stimulus for the bulk of the transmission will be determined by the output of the random number generator which utilizes both the DES encryption circuitry on the chip and the analog thermal noise inherent to the design.

In the simplified algorithm described above, when sampling the capacitance, all of the transmitting electrodes except for one were grounded and one electrode was driven with an input signal. Now each sampling of position will involve a measurement cycle which has several distinct portions to it. The system described below will be tamper proof enough to prevent someone from being able to externally determine what the present reading of the position sensor is. The only method of externally determining the present position sensor reading is through the encoded link. If someone moves the tag they will not be able to determine whether or not the tag is now in a new position which is equivalent to the old one unless they use the encoded link. The description of how the IC will measure its position is given below.

\section{Measurement cycle of sensor}

The main blocks of the more complicated sensor are shown in Figure 4. Each of the electrodes is connected to three switches. The switches will connect the electrode to one of the two D/A's or ground, according to commands from the digital logic. The A/D is used to measure the charge which is coupled onto the pickup electrode. This measures the capacitance of each of the electrodes to the fiducial.

Figure 5 shows an example of what the analog voltages would look like on the different points of the circuit during a portion of a position measurement cycle. The Figure shows four cycles of sampling of the capacitances in the position sensor.

During time period $t 0$, the digital logic determines the next random number to sequence the electrodes. Using this random number the digital logic then chooses (at random) two electrodes to be driven with a random amplitude. During this same time period ( 0 ), the analog electronics is settling out to an initial state of ground on all the electrodes and at the output of the gain block. This is done by having all switches open except for SGB, S1C, S2C, S3C, and S4C (refer to Figure 4).

During time $\mathrm{t} l$, the amplitude of the voltages which will drive the electrodes is loaded into the two D/A converters, D/A1 and D/A2. The two electrodes which are to be driven are connected to the D/A's by closing the appropriate switches. At time $t 1$, switches SGB, S1C, and S3C are opened then switches S1A and S3B are closed. This effectively applies a voltage across the coupling capacitance and couples charge into the gain block and generates the voltage at VGB. 
During time 2 , the voltage VGB is digitized by the A/D converter and the results are sent back to the digital logic for further analysis. At the end of $t 2$, all switches are opened again and the switches SGB, S1C, S2C, S3C, and S4C are closed (the same set as during time t0) and the three step cycle is repeated several thousand times during one measurement cycle. The major steps in the cycle are shown in Figure 6 for reference.

In Figure 5 the selection by the random number generator as far as the electrodes chosen for the D/A converters is as follows:

\begin{tabular}{|c|c|c|}
\hline $\begin{array}{c}\text { time } \\
\text { period }\end{array}$ & $\begin{array}{c}\text { electrode } \\
\text { D/A1 }\end{array}$ & $\begin{array}{c}\text { electrode } \\
\mathrm{D} / \mathrm{A} 2\end{array}$ \\
\hline $\mathrm{t} 0$ & 1 & 3 \\
\hline $\mathrm{t} 3$ & 3 & 3 \\
\hline $\mathrm{t} 6$ & 4 & 1 \\
\hline $\mathrm{t} 9$ & 2 & 4 \\
\hline
\end{tabular}

Note: The special case at time $\mathrm{t} 3$ has both $\mathrm{D} / \mathrm{A}$ 's pick the same electrode. The algorithm described below is used to pick the electrode driven by D/A2. Figure 5 shows that electrode 4 was chosen.

There is one slight complication when choosing which electrodes to use. The electrodes are chosen by two independent random bit patterns which each represent a number from 1 to 4 . Because the two numbers are independent of each other, approximately $25 \%$ of the time the two numbers will be the same. In this special case the random number would pick both of the D/A converters to drive the same electrode. This would cause several problems. If both electrode choices are the same, the chip will arbitrarily apply D/A1 to the electrode chosen and apply the output of D/A2 to a different electrode.

The methodology used for D/A2, when the two electrode numbers match is the following: an additional 2 bit counter within the IC, which counts from 1 to 3 (this means it has a count sequence $1,2,3,1,2,3,1, \ldots)$ will be incremented every time the two random numbers for electrode number match. The value chosen for the D/A2 will be the electrode number for $D / A 1$ added to the value of this new counter. The number of the electrode $\mathrm{D} / \mathrm{A} 2$ is then always different than the electrode chosen for $\mathrm{D} / \mathrm{A} 1$ and varies randomly with the input pattern.

\section{Selection of electrode driven and amplitude}

The random number generator produces a 64 bit random number each time the DES processes the input data. For each cycle, the IC uses a 20 bit portion of the 64 bit output word. The allocation of the bits is shown in Figure 7. These 20 bits of the random number are used to determine which of the electrodes is to be driven and the amplitude of the D/A converters, D/A1 and D/A2, which are both 8 bit D/A converters ( 7 bits plus sign). Figure 7 shows which two 8 bit bytes of the DES encrypted data are used to set the 
amplitudes of D/A1 and D/A2. It also shows which bits are used to determine the electrodes which are driven by the D/A converters. Of the 64 bit random number generated by the DES encryption, only 20 bits are used at a time. Because the position sensor only uses 20 bits out of the 64 bit pattern, one iteration of the random number generator can produce enough bits for 3 full cycles. After completion, there will be a wnit state in the analog circuitry to allow the random number generator to run a DES cycie on the previous random number. The random number generator will be described later in more detail.

\section{Qverall description of measurement cycle}

The position sensor has 3 main phases in the measurement cycle. The first phase is called the accumulation phase. Here the vast majority of the time is used and accumulated for the final position sensor reading. In order to simplify the final calculation phase, there is a second phase. The second phase begins when the accumulated values for the sensors are close to their final value. This second phase is the correction phase of the measurement. This phase modifies the drives to the sensors so that the accumulated registers will converge to their final desired values. When this phase is done, the calculation phase determines the present position. It transfers the results to the digital portion of the chip which then sends the position reading to the outside world.

\section{Accumulation of position readings}

This section describes the procedure used to accumulate the readings of the different driven electrodes and their relative coupling capacitances to the fiducial. Each of the 4 transmitting electrodes has a set of 5 registers as shown in Figure 8. At the end of a position sensor measurement cycle each electrode which is driven has its registers updated by the following algorithm (which is also flow charted in Figure 9). The chip will update only the register sets assigned to the electrodes driven during that one cycle. The drive for the driven electrodes is checked to see if it was positive or negative. If the drive was positive then the $A / D$ output is added to the accumulation register for that electrode and the drive amplitudes are added to the drive amplitude registers. If the drive was negative then the $A / D$ output is subtracted from the accumulation register for that electrode and the drive amplitudes for the driven electrodes are subtracted from the corresponding drive amplitude registers for that electrode.

At this point an example will help to illustrate why this particular algorithm was chosen. Figure 10 shows a small spreadsheet which illustrates 8 cycles of the algorithm in a setup where there are only 3 electrodes. The entries in A13, B13, and C13 are the relative capacitances from any one of the 3 electrodes to the fiducial. The columns $A$ and $C$ are the electrode numbers picked by a random number generator (any entries where the electrode numbers matched were discarded to simplify the spreadsheet). The columns B and $D$ are the respective amplitudes of the D/A outputs which were also chosen at 
random (and rounded to 2 places to simplify things). The column E corresponds to what the output of the A/D would read. For example line 3 has $A / D$ out $=0.93 * .51+$ $(-48)^{*} 0.85=0.0663$. The meaning of each of these numbers is as follows: the 0.93 is the magnitude of the voltage on D/A1. The 0.51 is the capacitance which D/A1 is coupled into the $A / D$ since it is driving electrode 1 . The $(-0.48)$ is the amplitude of $D / A 2$ and the 0.85 is the capacitance of the electrode that $\mathrm{D} / \mathrm{A} 2$ is driving (electrode 3 ). Also on line 3 it can be seen how the different registers are loaded (note here there are only 12 since it is a three electrode system, the actual chip will have 20 since it is a four electrode system). The two driven electrodes are electrode 1 (which uses the registers shown in column $\mathrm{F}$ through I) and electrode 3 (which uses the registers shown in columns $\mathrm{N}$ through $\mathrm{Q}$ ). Since the magnitude on electrode 1 is positive it is added into the registers $F$ through I and since the magnitude on electrode 3 was negative the numbers are subtracted from registers $\mathbf{N}$ through $\mathbf{Q}$.

The naming convention for the different columns $F$ through $Q$ is as follows: the columns which accumulate the A/D result (which corresponds to the bottom register in Figure 8) is labelled elnacc where $n$ is the electrode number. The rest of the columns are labelled elxy which is the yth register in the xth electrode set, i.e. el13 is a register in the set which accumulates measurements for electrode 1 . Since $x$ and $y$ are unequal ( 1 is not equal to 3 ) it is an interfering electrode which tends to corrupt the desired reading. The register el 13 measures electrode 3 crosstalk into the reading for electrode 1.

The net effect of this procedure of accumulating the readings is that all the registers elxy where $y=x$ are the sum of random variables which vary over the interval 0 to 1 with an average value of 0.5 while the registers elxy are the sum of rundom variables which vary over the interval of -1 to 1 with an average value of 0 . This results in the registers elxy where $x=y$ becoming large compared to the registers elxy where $x$ not $=y$. This can be seen in line 10 where el11, el22, and el33 are 4 to 10 times larger than the rest of the elxy registers. Already, with only 8 samples, a rough estimate of the capacitances can be made without correcting the output registers at all. The approximation is:

$$
\text { capacitance }=\text { elnacc } / \text { elnn }
$$

Using this approximation the following estimates are:

capacitance ell is $2.4519 / 3.54=0.69$ (not 0.51 )

capacitance el2 is $1.8085 / 1.85=0.98$ (not 0.95 )

capacitance el3 is $1.4178 / 2.05=0.69$ (not 0.85 )

The exact values of the different capacitances can be calculated by solving the three equations with three unknowns, which F10 through Q10 represent:

$$
\begin{aligned}
& \text { F10*cap1+G10*cap2+H10*cap3 }=\mathrm{I} 10 \\
& \mathrm{~J} 10 * \text { cap } 1+\mathrm{K} 10 * \text { cap2+L10*cap3 }=\mathrm{M} 10 \\
& \mathrm{~N} 10 * \text { cap } 1+\mathrm{O} 10 * \text { cap } 2+\mathrm{P} 10 * \text { cap3 } 3=\mathrm{Q} 10
\end{aligned}
$$


Since this takes an appreciable amount of hardware there is a different solution which is used on the IC. That solution involves careful adjustment of the drive amplitudes near the end of the 1 second measurement cycle. At the end of the cycle the values in F10, K10, and P10 will be the same large number and $\mathrm{G} 10, \mathrm{H} 10, \mathrm{J10}, \mathrm{L10}, \mathrm{N} 10$, and P10 are forced to zero. This procedure is described in the next section. The equations are then reduced to the following (where F10, K10, and P10 are forced to the final value, FV):

$$
\mathrm{FV}^{*} \text { cap1 } 110 \quad \mathrm{FV}^{*} \text { cap2=M10 } \quad \mathrm{FV}^{*} \text { cap3=Q10. }
$$

These equations needn't be solved further because the capacitances would just have a simple scaling constant of FV.

\section{Correction ohase of accumulation of position readings}

The final desired value of the accumulation registers, shown in Figure 8 (FV), is one register of each set contains all one's and the rest of the electrode drive amplitude registers of that set contain all zeroes. When the registers have reached these states, the answer is read out of the top bits of the accumulated A/D result register. Because of the random nature of the accumulated readings, the registers are not guaranteed to reach this set of states unless the algorithm is slightly modified.

The modification uses a different algorithm to set the amplitudes when the accumulated value for the drive register is near its final value. The first change in the algorithm is shown in Figure 11. There is an additional check which has been added. This will check to see if the driven electrode register will overflow if the new drive is added to the present accumulated value in the register. If the drive register would overflow, a bit is set for that particular electrode. This signals that the electrode register is close to the final value. This algorithm is followed until all four of the electrodes are within one conversion cycle of overflowing their final value. When all four electrodes are close to their final value (all four of the electrodes have set their bits referred to in Figure 11) the algorithm changes to the flowchart shown in Figure 12.

Figure 12 shows the procedure used to adjust all the registers to their final value. The algorithm sequences through all sets of electrode registers, one at a time. With each set of registers the IC does conversions to drive a pair of registers to their final value by doing a set of conversion cycles and only updating that pair of registers. This is then done for all of the registers in the chip.

The spreadsheet in Figure 13 shows a simple example which will help explain the procedure. The example uses the registers for the second driven electrode as indicated by the labelling of the columns in row 1 . To simplify the spreadsheet, the numbers shown are the deltas between the present values in the registers and the desired final values. The final value for all of the registers, except el22, is zero. The final value for el22 is all ones. 
The algorithm takes the first two electrodes (in this case el21 and el22) and drives them towards zero. Row 3 shows the first set of excitations applied to the electrodes. Since one of the two registers has a remaining offset of greater than 127 (el21) the amplitudes are determined by the random number generator. The signs of the excitations are determined by the present signs of the remainders left in the two registers el21 and el22. This type of sequencing is continued with the excitations shown on rows 5 and 7 . At row 8 the algorithm changes, since now both of the registers have amplitudes of less than 127 . The excitations applied to the two electrodes is just $(-1)$ times the values presently in the two registers el21 and el22. This last step then nulls out the first pair of registers in the set for electrode 2. The same procedure is then applied to the electrode el23 and el24 to finish off the register set. One thing to note is that on row 12 the sign in the register for el24 switches. This is because at the start of this sequencing the remainder in the el24 register is much less than the remainder in the el 23 register. The spreadsheet shows there is no problem caused by this. There is one slight complication. The final state for the driven electrodes has been called all ones. But actually the top bit is a zero and the remaining bits are all ones. The top bit in the register is a guard bit to prevent the register from overflowing during this operation.

\section{Random number sequencer}

The IC uses a sequence of random numbers to select the $\mathrm{D} / \mathrm{A}$ amplitudes and the driven electrodes during the analog position sensor reading. The IC will store the last random number generated after a position sensor reading is done. This number is used as the seed for the random number generator the next time a position sensor reading is requested. The very first time there is a request for position sensor reading the number read from the EEPROM is not random. The number will be randomized by the position sensor reading and a random number stored in the EEPROM. The first position sensor reading will be done during the initial testing of the chip. The chip is delivered for final assembly with a random number seed in the EEPROM.

The next random number is determined as follows. First the old random number is modified by replacing the bottom 8 bits with the last reading from the A/D converter. This randomizes the bottom 8 bits using the thermal noise floor caused by the high gain needed to detect the pickup signal. A single DES encryption is then run on the modified old random number. The result is a new 64 bit random number which is then divided into three 20 bit random numbers to be used for the next 3 conversion cycles. Four bits of the 64 are unused. The key used for the random number encryption/generation can arbitrarily be one of the secure encryption keys stored in EEPROM.

\section{Eiducial Pattern}

The main concern with the fiducial pattern is that the IC must have some portion of the fiducial directly above a pickup electrode. This is to maximize the received signal back into the IC from the transmitting electrode. Also, the conductive portion of the fiducial cannot completely cover the transmitting electrodes on the IC, to insure that movement of the IC with respect to the fiducial will cause a change in reading out of the position 
sensor. Figure 14 illustrates several orientations of a proposed fiducial located above an IC. Page 2 of Figure 14 shows two fiducials in poor orientations and provides an explanation. It should be noted here that the location, size and shape of the pickup electrodes at this time is not finalized and a different shape or size could be chosen to optimize the IC performance for a predetermined fiducial. 
TOP VIEW

(POLY NOT SHOWN)

SIDE VIEW

$\square \square \square \square \square \square \square \square \square \square \square \sim-$ Metal 2

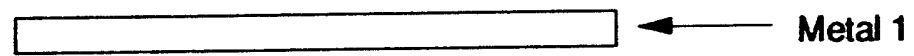

$\square \square \square \square \square \square \square \square \square \square \square \sim-$ Poly

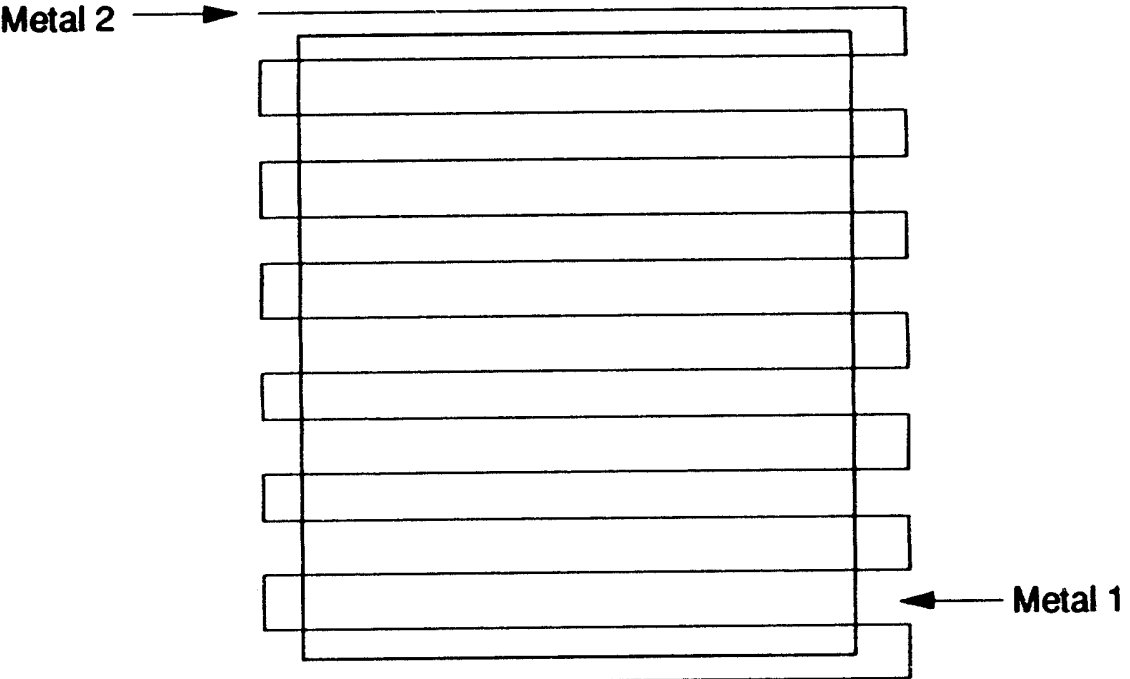

TAMPER SENSOR

FIGURE 1 


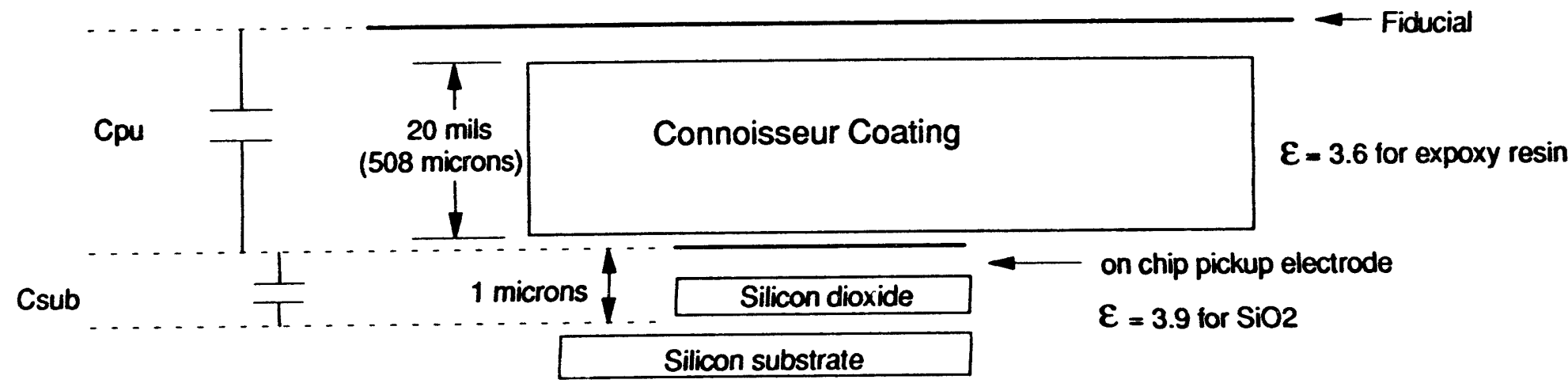

$\frac{C_{p u}}{\text { Csub }}=\frac{\varepsilon \text { connoisseur } / d \text { connoisseur }}{\varepsilon \text { silicon dioxide } / d \text { silicon dioxide }} \approx 500$

ON CHIP PICKUP ELECTRODE

FIGURE 2 


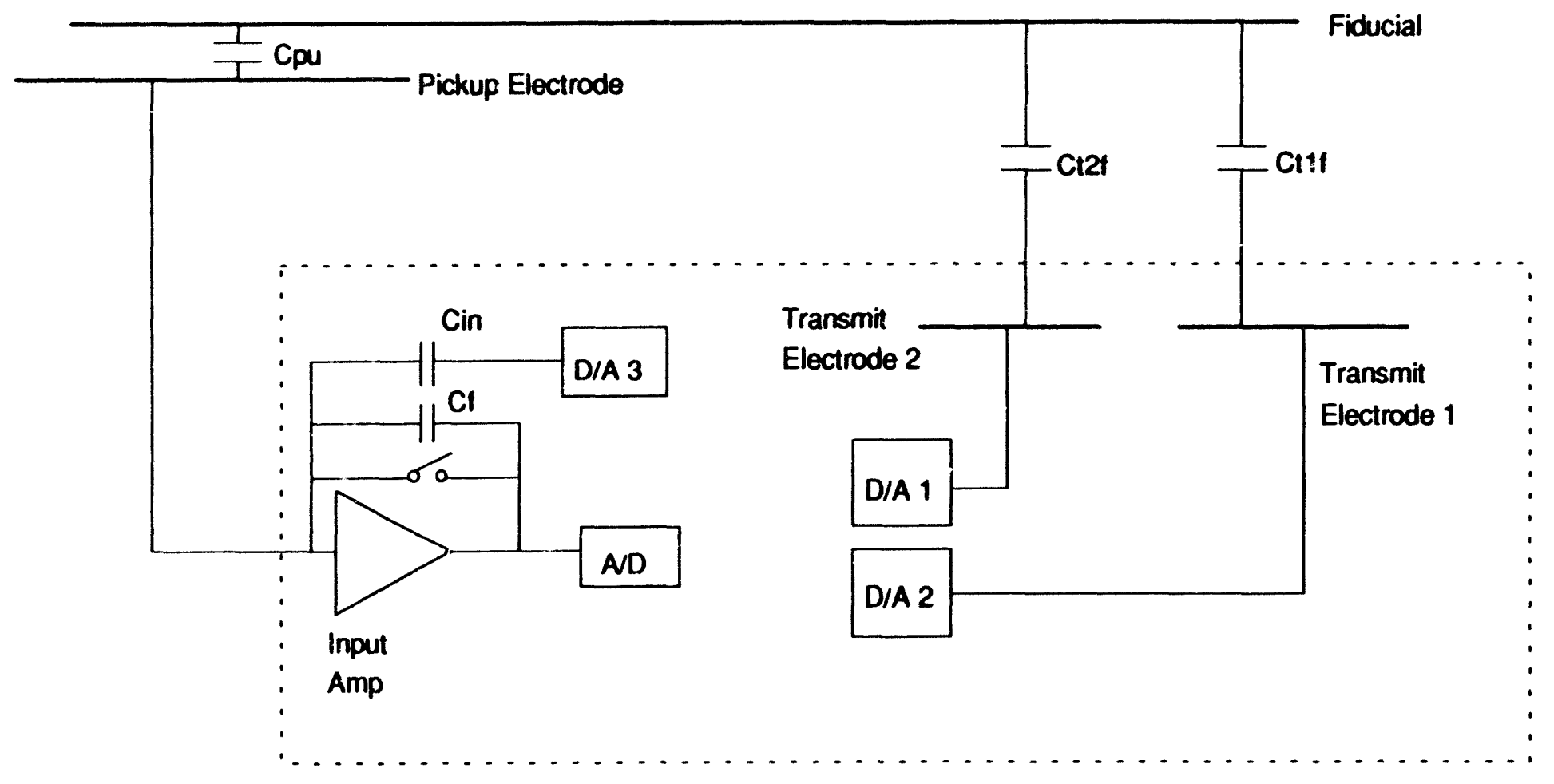

POSITION LOCATION SENSOR

FIGURE 3 
Fiducial

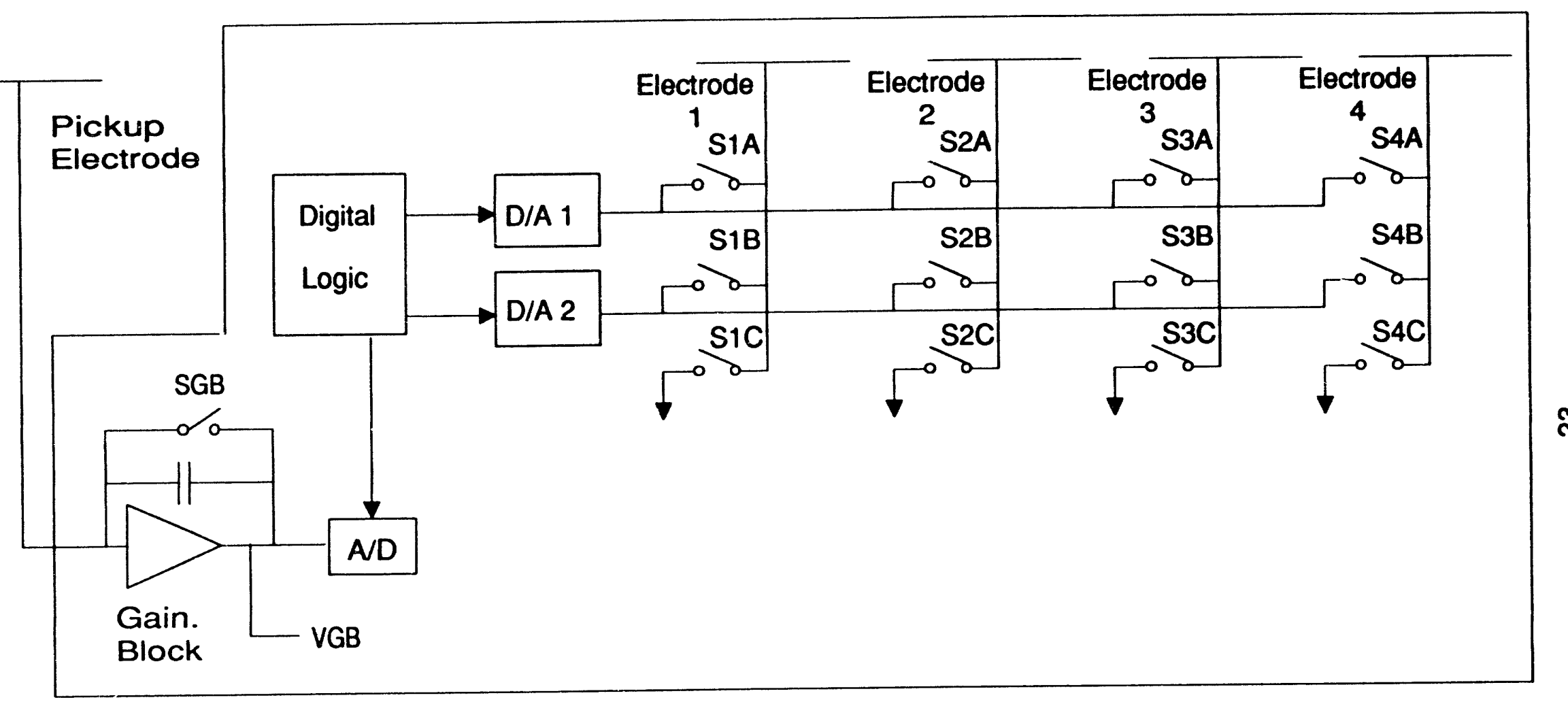

ANALOG PORTION OF SENSOR

FIGURE 4 


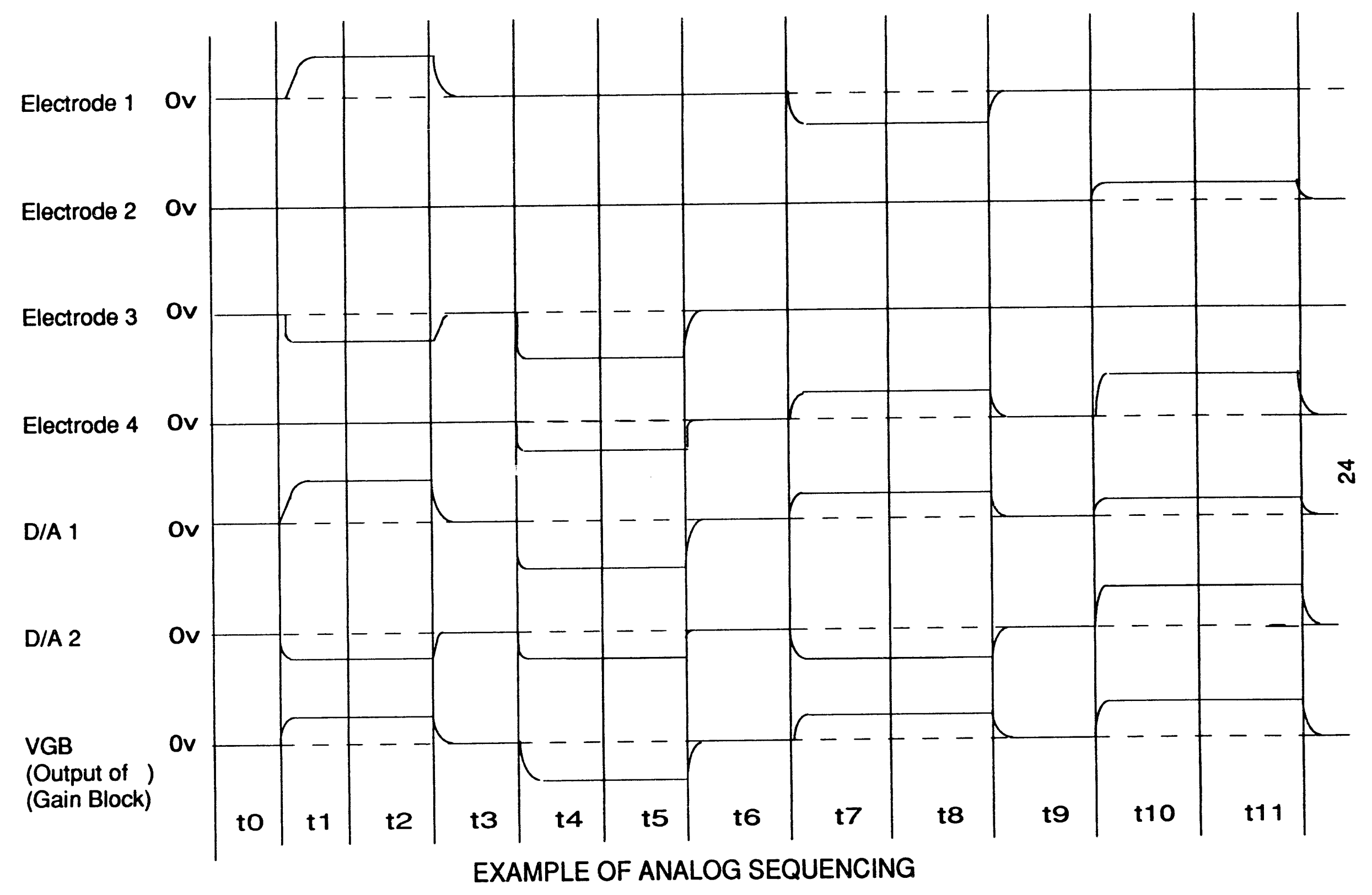

FIGURE 5 


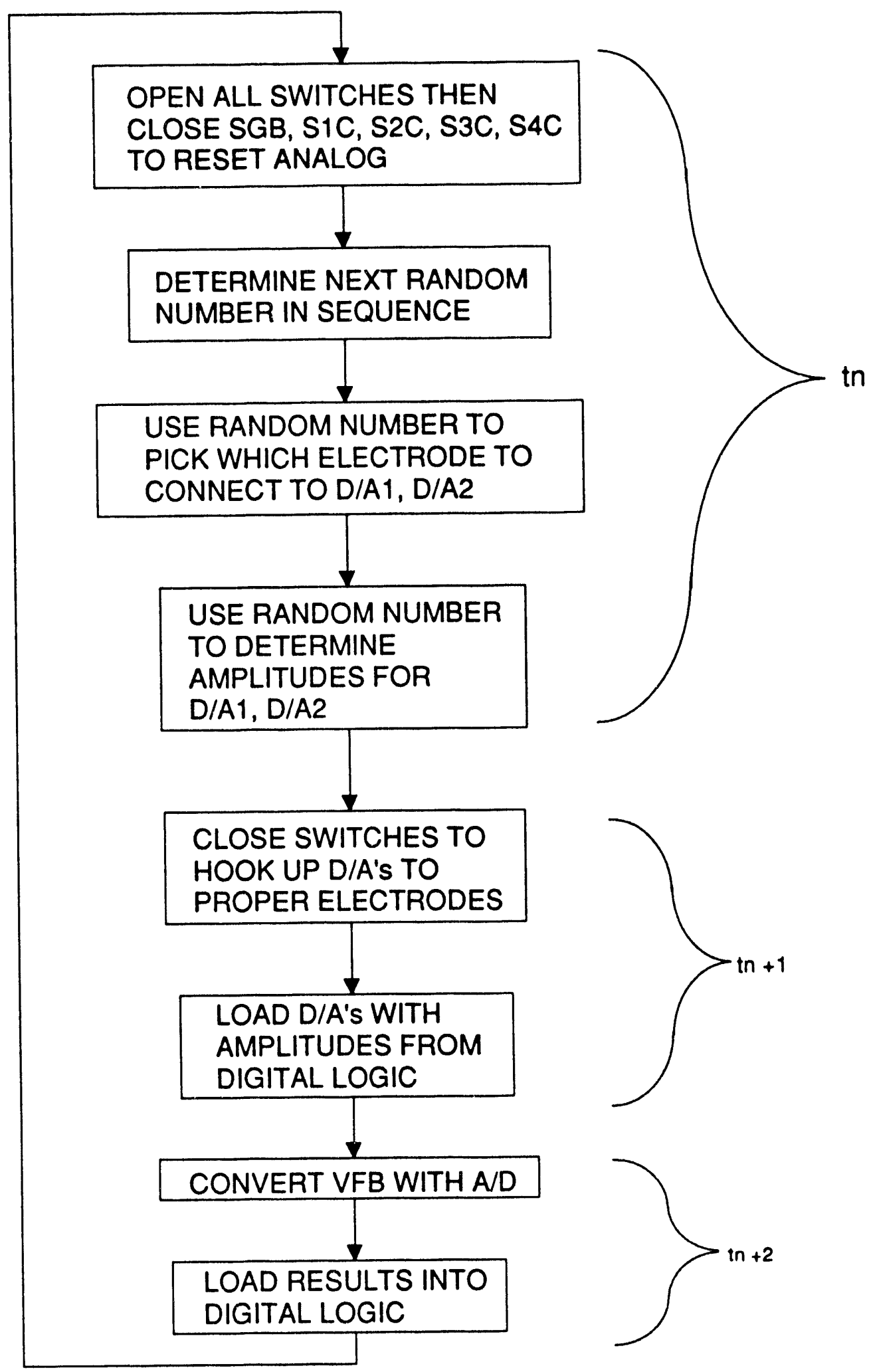

ONE CYCLE OF POSITION SENSOR MEASUREMENT

FIGURE 6

25 
ENCRYPTED DES OUTPUT (For each 20 bit chunk)

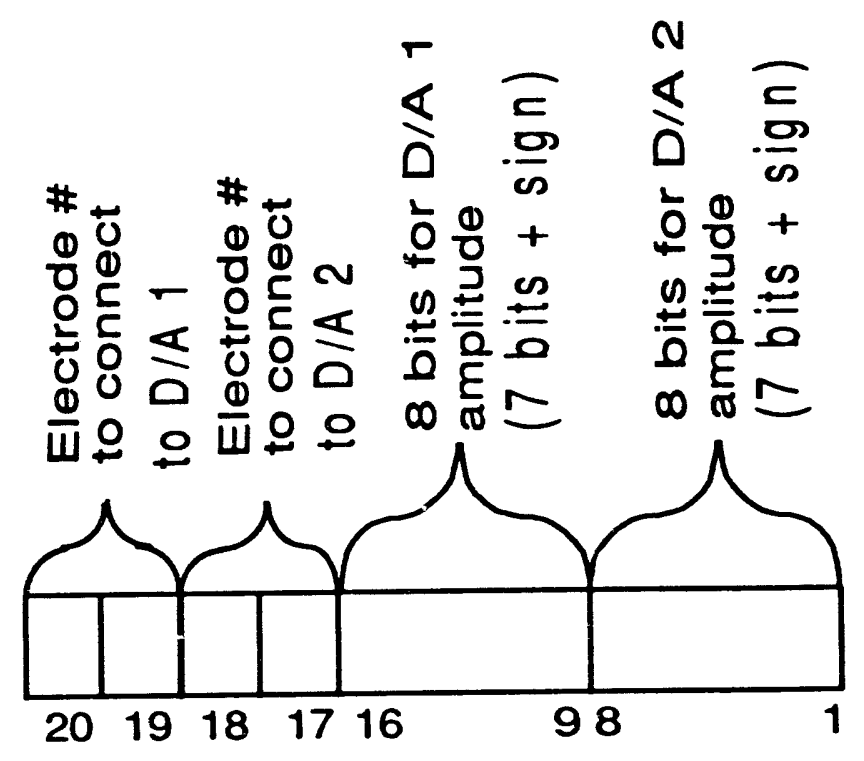

\begin{tabular}{c|c|c} 
bit & bit & $\begin{array}{c}\text { electrode \# } \\
\text { for D/A 1 }\end{array}$ \\
\hline 0 & 0 & 1 \\
\hline 0 & 1 & 2 \\
\hline 1 & 0 & 3 \\
\hline 1 & 1 & 4 \\
\hline
\end{tabular}

\begin{tabular}{c|c|c} 
bit & bit & electrode \# \\
18 & 17 & for D/A 2 \\
\hline 0 & 0 & 1 \\
\hline 0 & 1 & 2 \\
\hline 1 & 0 & 3 \\
\hline 1 & 1 & 4 \\
\hline
\end{tabular}

*Special case if bit $20=$ bit 18 and bit $19=$ bit 17

D/A 2 is determined per text 


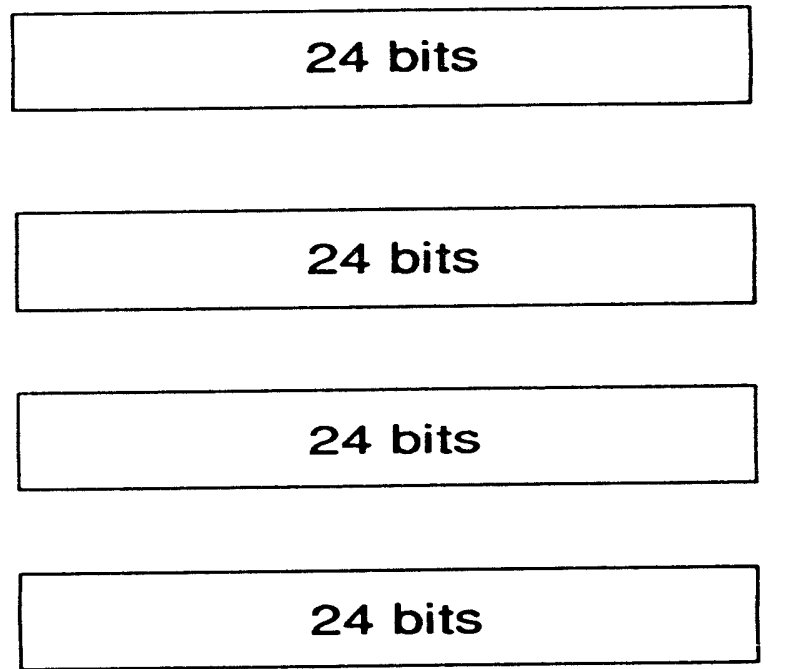

24 bits

28 bits
Electrode 1 drive amplitudes

Electrode 2 drive amplitudes

Electrode 3 drive amplitudes

Electrode 4 drive amplitudes

Accumulated AVD result

ACCUMULATION FOR REGISTERS FOR EACH ELECTRODE

FIGURE 8 


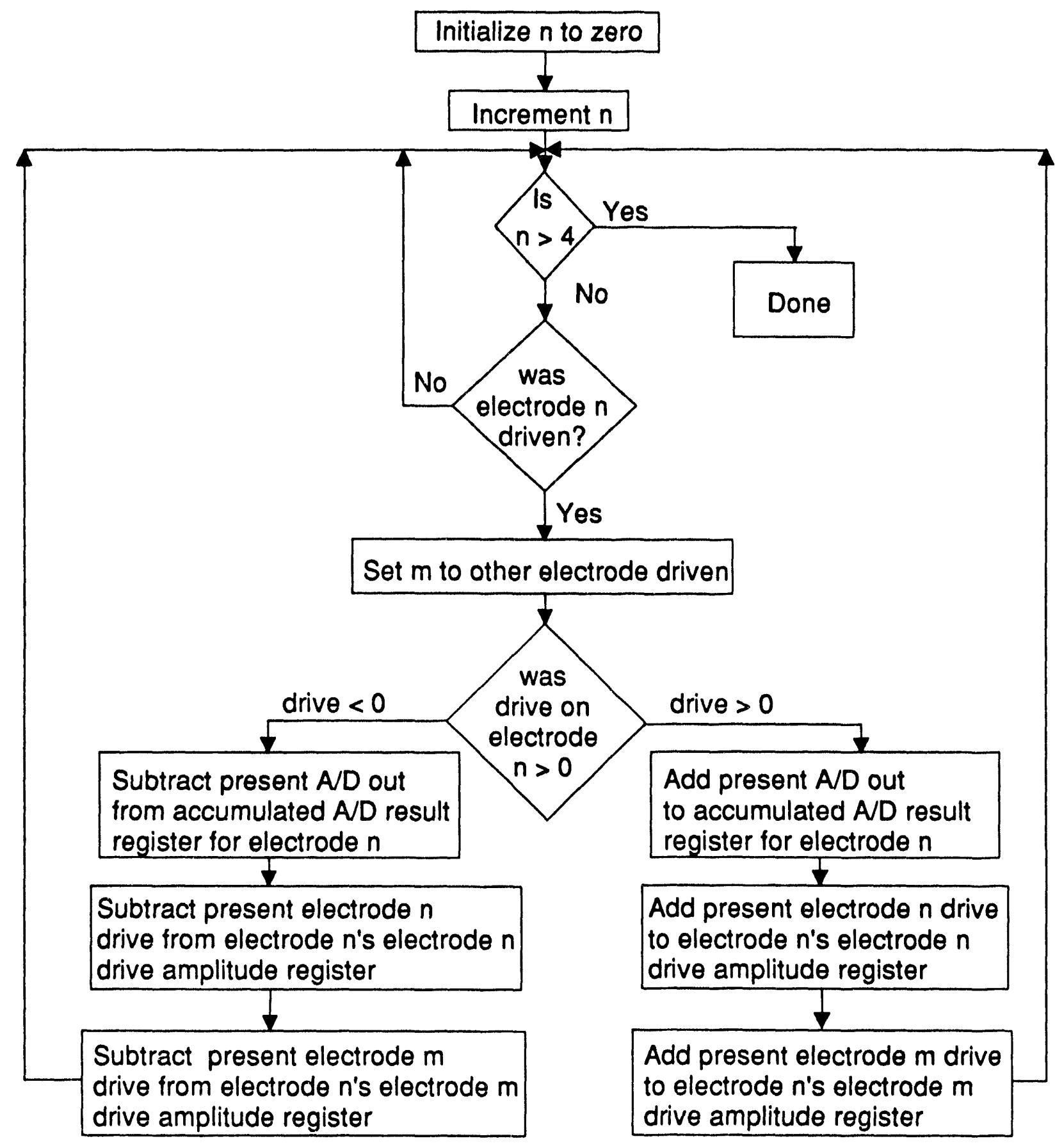

FLOW CHART OF ACCUMULATION OF MEASUREMENT CYCLES

FIGURE 9 


\begin{tabular}{|c|c|c|c|c|c|c|c|c|c|c|c|c|c|c|c|c|c|}
\hline & A & B & C & $\bar{D}$ & $\overline{\mathbf{E}}$ & $F$ & G & H & 1 & $\mathrm{~J}$ & $\mathrm{~K}$ & $\mathbf{L}$ & $M$ & $\mathbf{N}$ & 0 & $\mathbf{P}$ & $\mathbf{Q}$ \\
\hline 1 & $\mathrm{~d} / \mathrm{a} 1$ & d/a 1 & $d / a_{2}$ & $d / a 2$ & AD out & el 11 & el 12 & el 13 & el1 acc e & $\mathrm{el} 21$ & $\mathrm{el} 22$ & $\mathrm{el} 23$ & el2acc & el 31 & el 32 & el 33 & el3acc \\
\hline 2 & el \# & mag & el \# & mag & & & & & & & & & & & & & \\
\hline 3 & 1 & 0.93 & 3 & -0.48 & 0.0663 & 0.93 & q & -0.48 & 0.0663 & 9 & 0 & 0 & d & -0.93 & q & 0.48 & -0.0663 \\
\hline 4 & 2 & -0.37 & 1 & 0.59 & -0.0506 & 1.52 & -0.37 & -0.48 & 0.0157 & -0.59 & 0.37 & q & 0.0506 & -0.93 & $q$ & 0.48 & -0.0663 \\
\hline 5 & 1 & -0.41 & 2 & -0.11 & -0.3136 & 1.93 & -0.26 & -0.48 & 0.3293 & -0.18 & 0.48 & o & 0.3642 & \begin{tabular}{|c|}
-0.93 \\
\end{tabular} & 0 & 0.48 & -0.0663 \\
\hline 6 & 3 & 0.06 & 2 & -0.17 & -0.1105 & 1.93 & -0.26 & -0.48 & 0.3293 & -0.18 & 0.65 & -0.06 & 0.4747 & -0.93 & -0.17 & 0.54 & \begin{tabular}{|l|}
-0.1768 \\
\end{tabular} \\
\hline 7 & 2 & -0.69 & 1 & -0.33 & \begin{tabular}{|l|}
-0.8238 \\
\end{tabular} & 2.26 & 0.43 & -0.48 & 1.1531 & 0.15 & 1.34 & -0.06 & 1.2985 & -0.93 & \begin{tabular}{|c|}
-0.17 \\
\end{tabular} & 0.54 & -0.1768 \\
\hline 8 & 2 & 0.51 & 1 & 0.05 & 0.51 & 2.31 & 0.94 & -0.48 & 1.6631 & 0.2 & 1.85 & -0.06 & 1.8085 & -0.93 & \begin{tabular}{|l|}
-0.17 \\
\end{tabular} & 0.54 & -0.1768 \\
\hline 9 & 1 & 0.92 & 3 & 0.85 & 1.1917 & 3.23 & 0.94 & 0.37 & 2.8548 & 0.2 & 1.85 & -0.06 & 1.8085 & -0.01 & -0.17 & 1.39 & 1.0149 \\
\hline 10 & 1 & 0.31 & 3 & -0.66 & -0.4029 & 3.54 & 0.94 & -0.29 & 2.4519 & 0.2 & 1.85 & -0.06 & 1.8085 & -0.32 & -0.17 & 2.05 & 1.4178 \\
\hline 11 & caps & & & & & & & & & & & & & & & & \\
\hline 12 & el1 & $e l 2$ & $\mathrm{e} 13$ & & & & & & & & & & & & & & \\
\hline 13 & 0.51 & 0.95 & 0.85 & & & & & & & & & & & & & & \\
\hline
\end{tabular}

Figure 10 


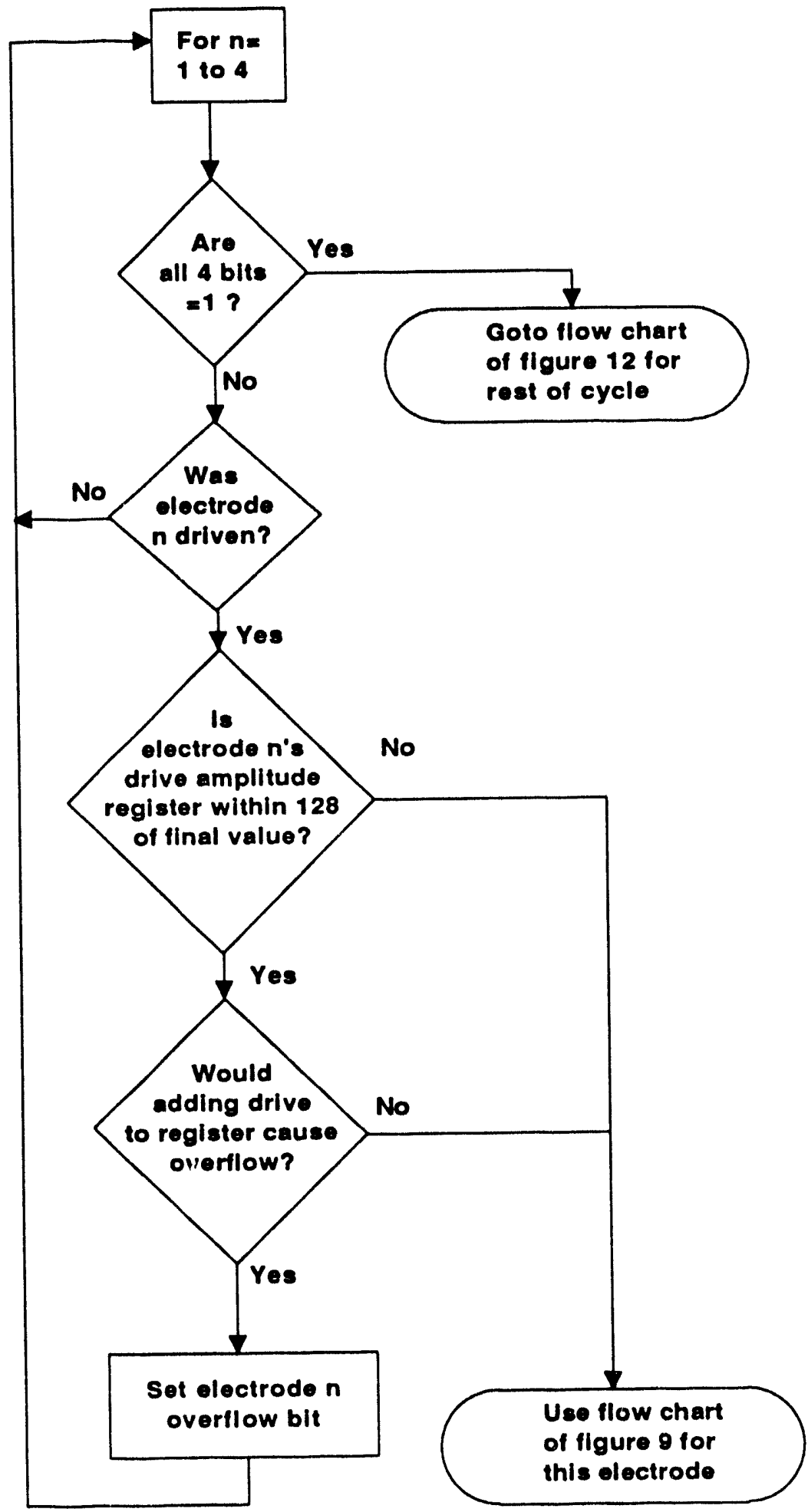

Transitional cycles

Figure 11 


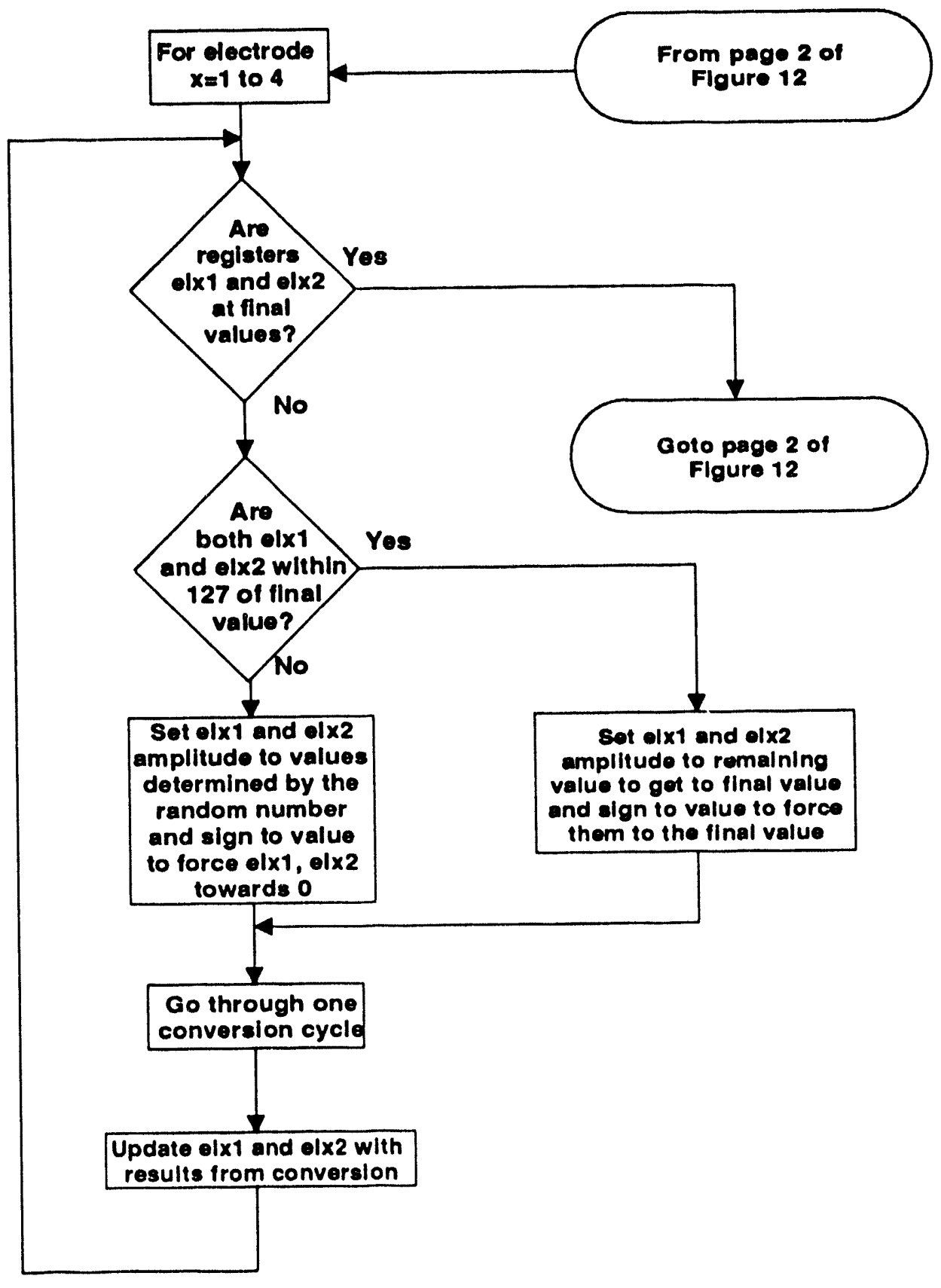

Final adjustment cycle Figure 12

page 1 of 2 


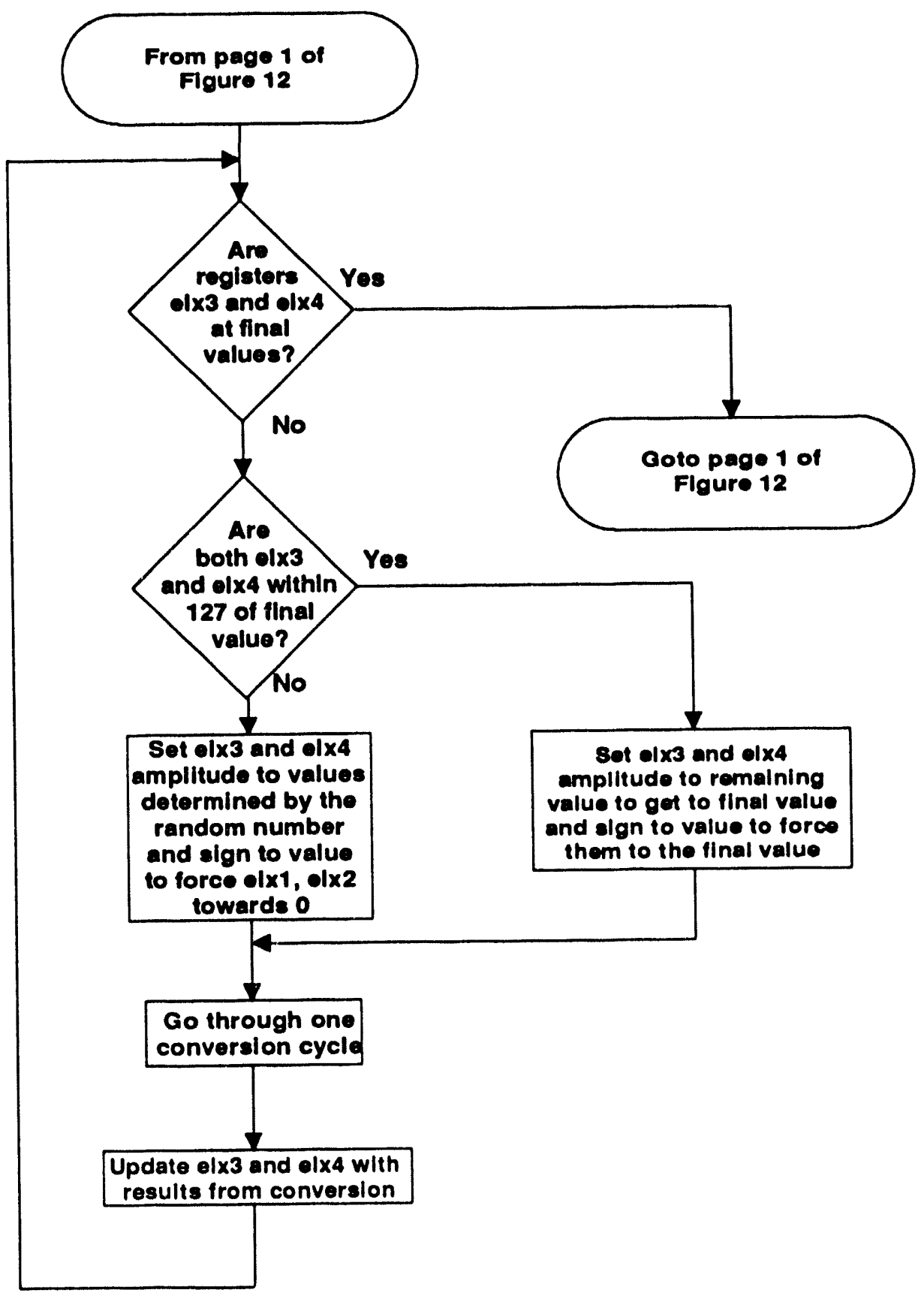

Final adjustment cycle

Figure 12

page 2 of 2 


\begin{tabular}{|r|r|r|r|r|}
\hline & \multicolumn{1}{|c|}{ A } & \multicolumn{1}{c|}{ B } & \multicolumn{1}{c|}{ C } & \multicolumn{1}{c|}{ D } \\
\hline 1 & el21 & el22 & el23 & el24 \\
\hline 2 & 327 & -115 & 143 & -10 \\
\hline 3 & -91 & 20 & 0 & 0 \\
\hline 4 & 236 & -95 & 143 & -10 \\
\hline 5 & -69 & 45 & 0 & 0 \\
\hline 6 & 167 & -50 & 143 & -10 \\
\hline 7 & -98 & 29 & 0 & 0 \\
\hline 8 & 69 & -21 & 143 & -10 \\
\hline 9 & -69 & 21 & 0 & 0 \\
\hline 10 & 0 & 0 & 143 & -10 \\
\hline 11 & 0 & 0 & -63 & 39 \\
\hline 12 & 0 & 0 & 80 & 29 \\
\hline 13 & 0 & 0 & -80 & -29 \\
\hline 14 & 0 & 0 & 0 & 0 \\
\hline
\end{tabular}

Figure 13 

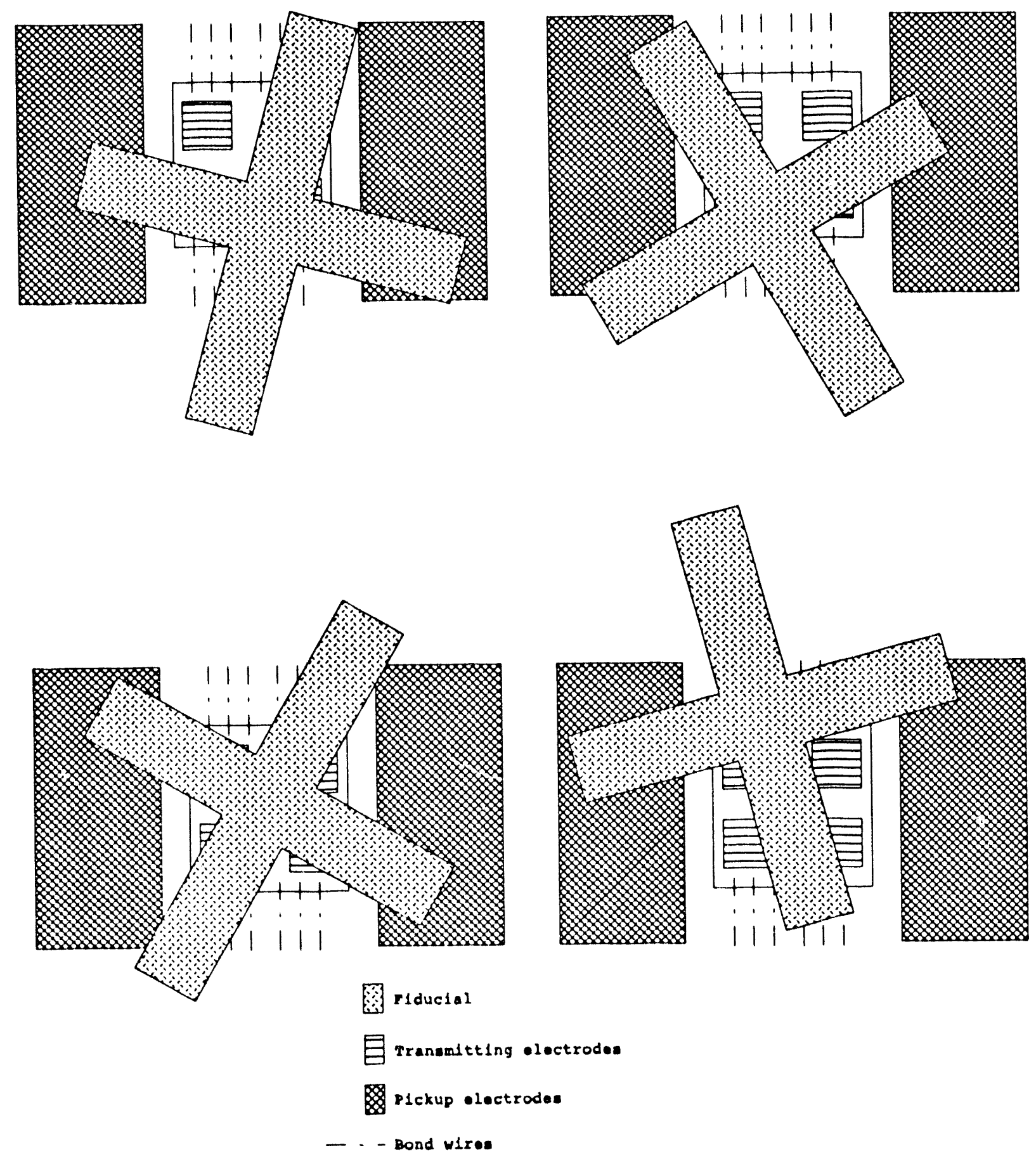

Figure 14 page 1 of 2

Examples of a good fiducial pattern 


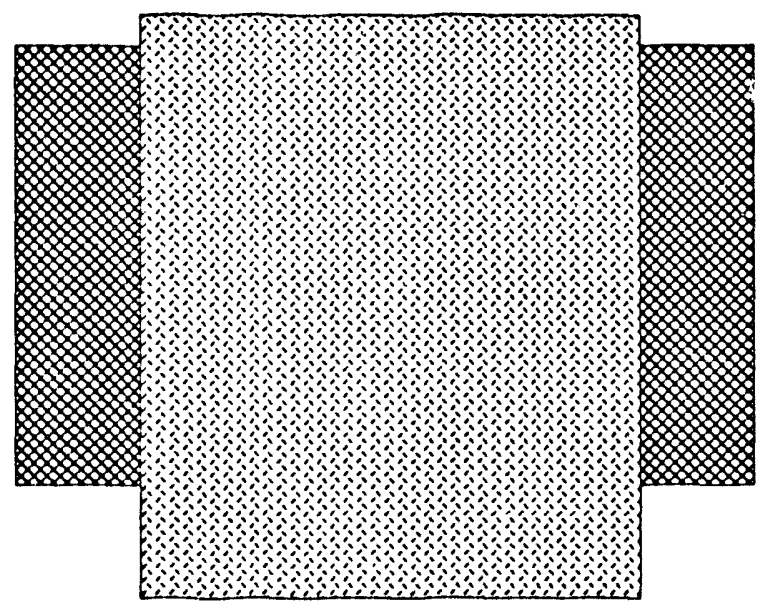

Movements in fiducial

will not cause changes in position reading

Fiducial will not couple into the pickup electrode

Figure 14 page 2 of 2

Examples of poor fiducial patterns 

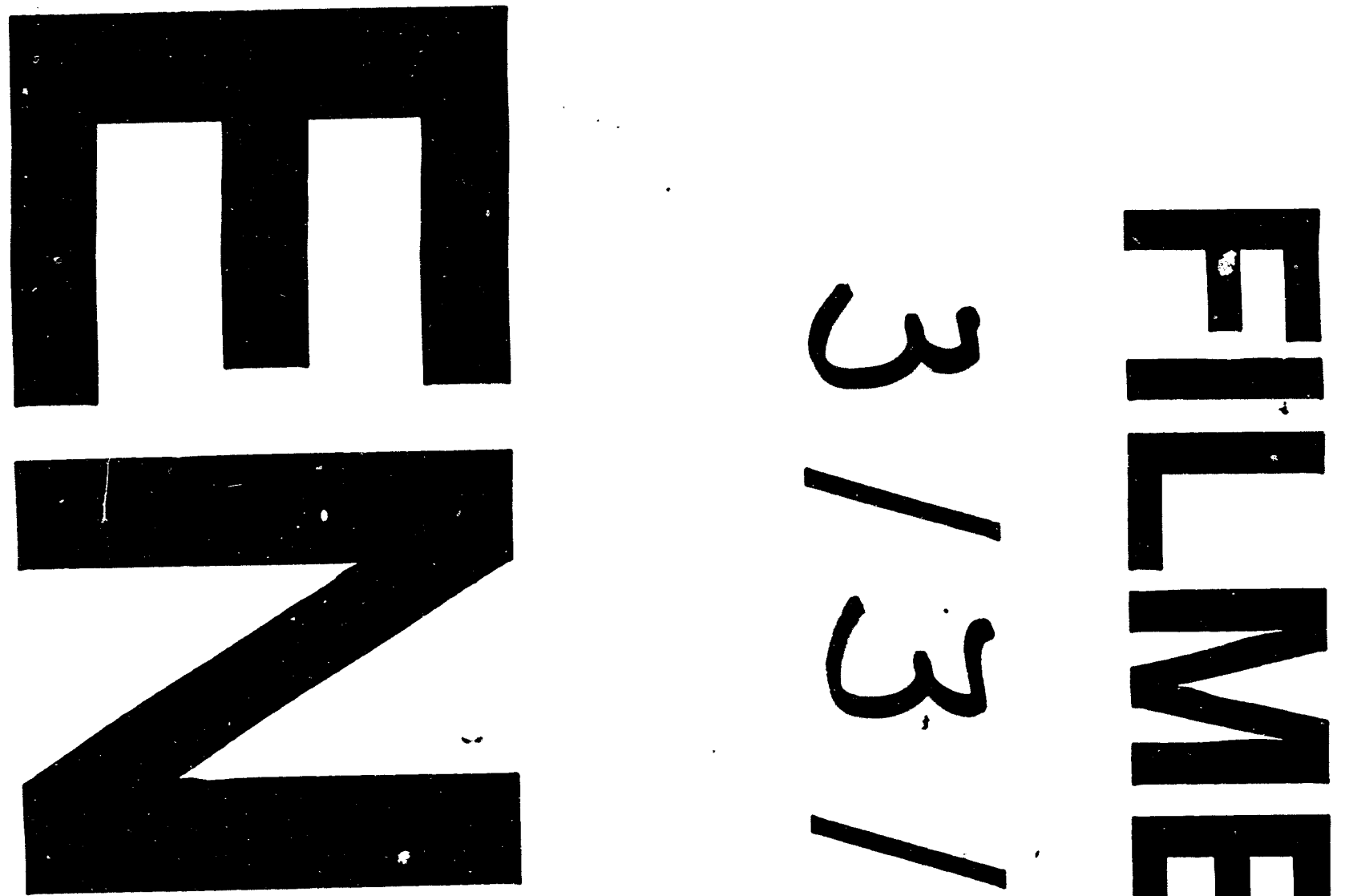

W
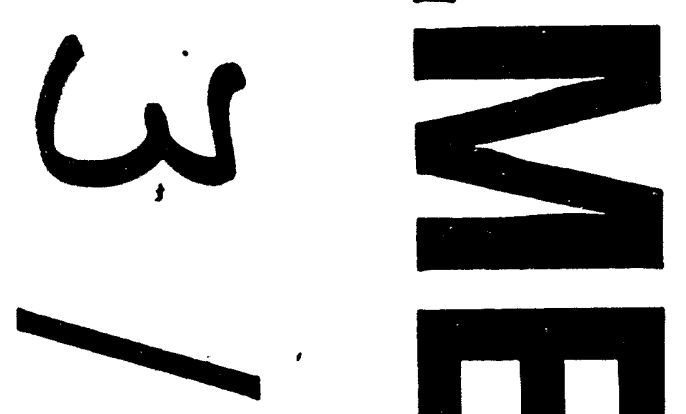

0
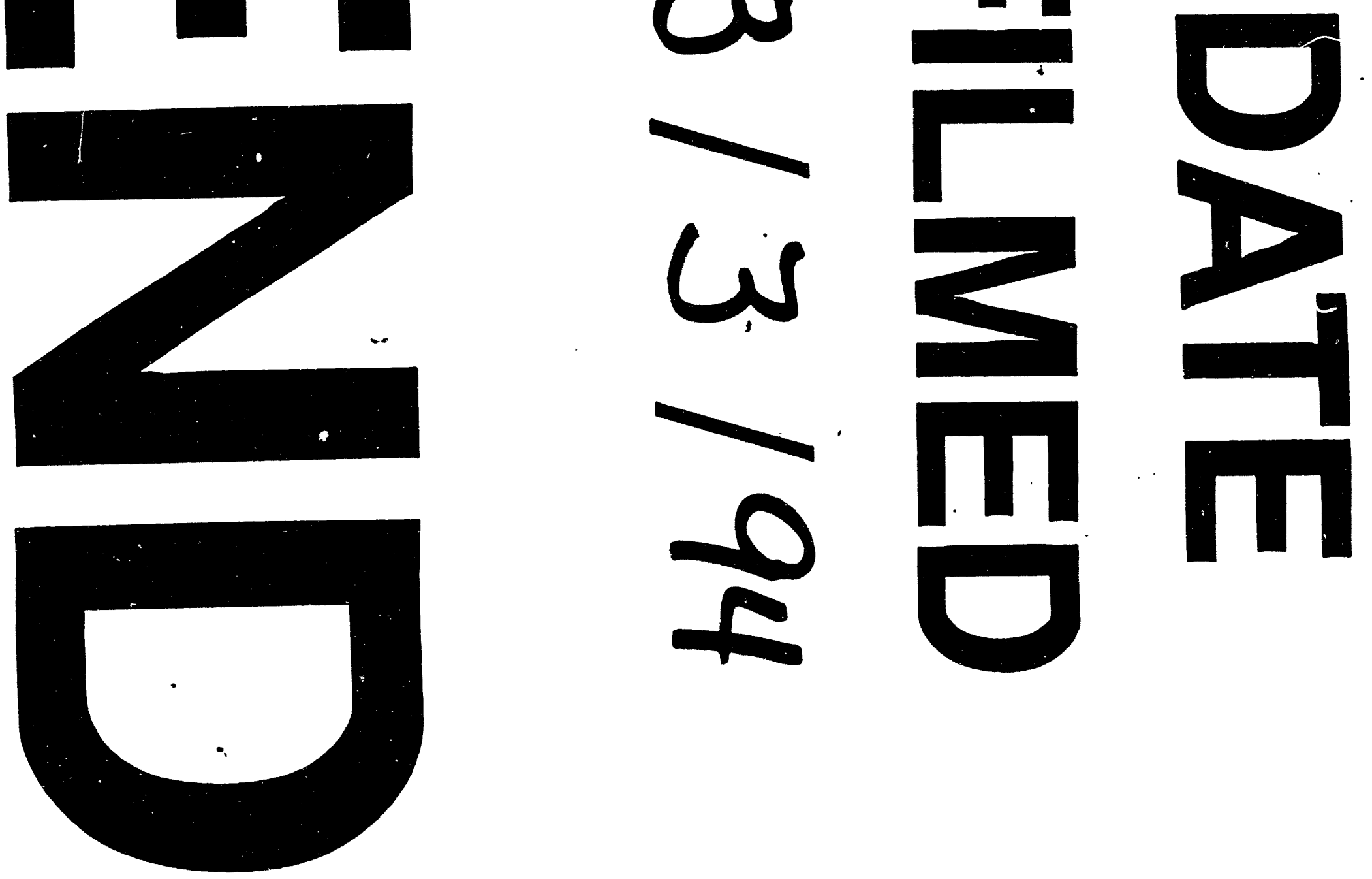
